\title{
The Effectiveness of Self-Management Interventions for Individuals with Low Health Literacy and/or Low Income: A Descriptive Systematic Review
}

\author{
Jamie Schaffler, RN, BSc, MSc $(N)^{7}$, Katerina Leung, $R N, B S c, M S c(N)^{7}$, \\ Sarah Tremblay, RN, BSc, MSc $(N)^{7}$, Laura Merdsoy, $R N, B A, M S c(N)^{7}$, Eric Belzile, $M S c^{2}$, \\ Angella Lambrou, MLIS ${ }^{3}$, and Sylvie D. Lambert, RN, BSC(N), $P h D^{1,2}$ \\ 'Ingram School of Nursing, McGill University, Quebec, Montreal, Canada; ${ }^{2}$ St. Mary's Research Centre, Quebec, Montreal, Canada; ${ }^{3}$ Schulich \\ Library of Science and Engineering, McGill University, Quebec, Montreal, Canada.
}

\begin{abstract}
BACKGROUND: With the burden of chronic illness increasing globally, self-management is a crucial strategy in reducing healthcare costs and increasing patient quality of life. Low income and low health literacy are both associated with poorer health outcomes and higher rates of chronic disease. Thus, self-management represents an important healthcare strategy for these populations. The purpose of this study is to review self-management interventions in populations with low income or low health literacy and synthesize the efficacy of these interventions. METHODS: A systematic review of trials evaluating the efficacy of self-management interventions in populations with low income or low health literacy diagnosed with a chronic illness was conducted. Electronic databases were primarily searched to identify eligible studies. Data were extracted and efficacy summarized by self-management skills, outcomes, and content tailoring.
\end{abstract}

RESULTS: 23 studies were reviewed, with ten reporting an overall positive effect on at least one primary outcome. Effective interventions most often included problemsolving as well as taking action and/or resource utilization. A wide range of health-related outcomes were considered, were efficacious empowerment and diseasespecific quality of life were found to be significant. The efficacy of interventions did not seem to vary by duration, format, or mode of delivery or whether these included individuals with low health literacy and/or low income. Tailoring did not seem to impact on efficacy.

DISCUSSION: Findings suggest that self-management interventions in populations with low income or low health literacy are most effective when three to four selfmanagement skills are utilized, particularly when problem-solving is targeted. Healthcare providers and researchers can use these findings to develop education strategies and tools for populations with low income or low health literacy to improve chronic illness selfmanagement.

Electronic supplementary material The online version of this article (https://doi.org/10.1007/s11606-017-4265-x) contains supplementary material, which is available to authorized users.

Received November 3, 2017

Revised November 20, 2017

Accepted December 4, 2017

Published online February 9, 2018
KEY WORDS: self-management; chronic disease; disease management; vulnerable populations; health literacy.

J Gen Intern Med 33(4):510-23

DOI: $10.1007 / \mathrm{s} 11606-017-4265-\mathrm{x}$

(C) Society of General Internal Medicine 2018

\section{BACKGROUND}

Currently, $50 \%$ of Americans ${ }^{1}$ and $60 \%$ of Canadians ${ }^{2}$ are diagnosed with a chronic condition, with an increasing number of patients suffering from multi-morbidities. ${ }^{3}$ The prevalence of chronic disease and the long-term follow-up required in chronic disease management are resulting in a substantial economic burden on the healthcare system. ${ }^{4}$ To assess and improve chronic disease care and control healthcare costs, the Chronic Care Model (CCM) was developed, emphasizing six essential elements in the management of chronic illnesses: healthcare organization, linkages to community resources, delivery system design, clinical decision support, clinical information systems, and self-management support. ${ }^{5}$ Several reviews have found that implementation practices in accordance with the CCM improve the quality of care and health outcomes for patients ${ }^{6,7}$ as well as reduce healthcareassociated costs. ${ }^{7,8}$ Of the six CCM elements, selfmanagement support is the most commonly implemented and has received the most attention. ${ }^{7}$

Self-management refers to the tasks that an individual must undertake to live well with a chronic condition. ${ }^{9}$ It includes five core skills: (1) decision-making, (2) problem-solving, (3) utilizing resources, (4) forming a client-healthcare provider partnership, and (5) taking action. ${ }^{10}$ Systematic reviews have shown that self-management interventions have mixed efficacy across chronic illnesses, ${ }^{11-15}$ with positive outcomes particularly noted for symptom management, pain control, and role functioning. ${ }^{10,16}$ This research suggests that both the type of chronic illness and the nature of the self-management skills utilized mediate the efficacy of the interventions. ${ }^{17,18} \mathrm{Al}$ though most studies have been conducted with populations of females with higher socioeconomic status (SES) and education, ${ }^{19,20}$ in the past 2 decades, there has been increased 
attention given to particular vulnerable subgroups, including those of low income or low health literacy.

Education and income are key factors in determining health. ${ }^{21}$ The United States Census Bureau reports that 46.7 million Americans are affected by poverty. ${ }^{22}$ This is significant because chronic illnesses and lower life expectancies are more prevalent in low-income populations regardless of age, gender, race, and geographical location. ${ }^{23}$ Lifestyle factors including limited access to affordable housing, healthy foods, and recreational facilities, as well as increased stress levels, may contribute to these unfavorable health outcomes. ${ }^{24}$ Further, low-income populations may struggle with limited access to healthcare. ${ }^{24}$

Additionally, low levels of education are associated with poorer health, more stress, and lower self-efficacy. ${ }^{25}$ Individuals with low education tend to have limited socioeconomic mobility, lower incomes, insecure employment, and poorer working conditions, all of which contribute to adverse health outcomes. ${ }^{26}$ Importantly, low education tends to decrease overall literacy and health literacy levels, which in turn adversely impact on the development self-management skills. ${ }^{26}$ It is estimated that $36 \%$ of adults in the US and $60 \%$ in Canada have limited health literacy. ${ }^{27,28}$ This has been associated with decreased medication adherence and use of preventative services, higher rates of hospitalizations and mortality, and overall poorer health outcomes. ${ }^{4}$

Thus, interventions for improving self-management skills among a population of low-income or low health literate patients may have profound effects on health outcomes. To our knowledge, the present review is the first one to describe the current use of self-management interventions in patients with low income and/or low health literacy and synthesize the efficacy of these interventions by self-management skills, health-related outcomes, and extent of content tailoring.

\section{METHODS}

The Preferred Reporting Items for Systematic Reviews and Meta-Analyses (PRISMA) statement was used ${ }^{29}$ to conduct this descriptive systematic review of the literature (checklist included in the Electronic Supplementary Material S1).

\section{Criteria for Considering Studies}

Types of Studies and Participants. Eligible studies were published peer-reviewed experimental or quasi-experimental trials in which: (1) a group of adults with a chronic physical illness (with or without a comorbid mental illness) of low income and/or low health literacy received a selfmanagement intervention and were compared with a group who did not receive the intervention; and (2) outcomes were measured pre- and post-intervention. Low income and low health literacy were defined as per study authors. Only English and French full texts were included, and studies dating more than 15 years ago were excluded.

Types of Interventions. Studies included were those that evaluated an intervention aimed at enhancing one or more of the five aforementioned core self-management skills. ${ }^{9}$ Restrictions were not applied to the mode of delivery or to the type of care setting. However, solely pharmacological interventions were excluded. Interventions targeting minority groups were also excluded to decrease potential confounding factors.

Types of Outcomes. The outcomes of interest included physiological, behavioral, and/or psychosocial outcomes. Other outcomes of interest included illness knowledge, financial, and healthcare service utilization. Therefore, studies were included if quantitative data were obtained for at least one relevant outcome and if statistical methods were used to evaluate the differences between control and intervention groups.

\section{Search Methods}

Eligible studies were identified through an electronic search of MEDLINE, PsycINFO, CINAHL, and Embase. The search strategy used a combination of keywords and medical subject heading (MeSH) terms related to low income, low health literacy, self-management, and the study design (see Electronic Supplementary Material S2). All titles were downloaded to EndNote. Hand searches were performed on the reference lists of all included manuscripts to identify other eligible studies. At least two authors independently assessed the initial eligibility of the retrieved titles and abstracts. The full-texts of all eligible citations were independently examined by at least three authors to confirm eligibility. Any disagreements were discussed at regular team meetings until a consensus was reached.

\section{Data Collection}

A standardized form based on the Cochrane Handbook for Systematic Reviews of Interventions was used to extract da$\mathrm{ta}^{30}$ from each manuscript (e.g., citation details, study design, inclusion criteria, sample size, population characteristics, selfmanagement skills targeted). Two authors independently extracted the data, and disagreements were discussed and resolved with a third author.

The methodological quality of included studies was assessed by five authors using the following criteria $^{30,31}$ : control group, $n>25$ per group, sufficient power to detect moderate effect, inclusion criteria specified, assessment of reliability and validity of measures, adequate sequence generation, allocation concealed, blind assessment, intention to treat used, $>80 \%$ of the sample in the final analysis, and reasons for attrition stated. 
Each criterion was scored as either a $1=$ yes or $0=$ no. A high methodological quality was defined as a score of at least 9 moderate methodological quality between 6 and 8 , and low quality as scores of $\leq 5$.

\section{Data Synthesis}

As there was substantial heterogeneity across studies in study design, demographics, type of interventions, and outcomes, a descriptive synthesis was conducted as opposed to a metaanalysis. $^{32}$ Date extracted were summarized by one author and verified by at least one other author. Efficacy of the interventions was summarized based on self-management skills, outcomes, and integration of individualized assessment (tailoring). ${ }^{33}$ For each outcome, efficacy was based on statistical significance $(p<0.05)$ and a Cohen's effect size $(\mathrm{ES})$ of at least $\mathrm{d}=0.2$ or odds ratio $(\mathrm{OR})>1.44 .^{34}$ For studies lacking sufficient data to calculate an ES, only statistical significance was considered. For the analysis by self-management skills and tailoring, an intervention was said to be effective, if the results were significant for at least one primary outcome. For the analysis by outcome, if at least $50 \%$ of the analyses were positive, the conclusion was that the interventions were effective for that outcome.

\section{RESULTS}

The electronic search identified 2976 titles, whereby 50 manuscripts underwent full-text review. Of these, 27 were subsequently excluded, resulting in 23 studied included in the present review. Figure 1 outlines the systematic review process.

\section{Overview of Studies}

Types of Studies and Participants. Table 1 summarizes the characteristics of the 23 studies included. Most studies compared two groups, ${ }^{35,37-40,42-47,49,50,52,54,55,57}$ and all studies were conducted in the US, with sample sizes ranging from $28^{47}$ to $5457 .{ }^{36}$ Participants were predominately female aged 40-68 diagnosed with diabetes $(n=9)$ or hypertension $(n=3)$. The majority of the studies examined the efficacy of selfmanagement interventions in a population of low-income participants exclusively $(n=13)$. Seven studies examined the efficacy in participants with low health literacy. Three studies included participants with both low health literacy and low income.

Low income defined. Most studies operationalized low income by stating that participants were recruited from healthcare facilities that were "federally qualified heath centers" $(n=4),{ }^{36,37,41,44}$ "safety-net" clinics $(n=3),{ }^{35,38,55}$ or

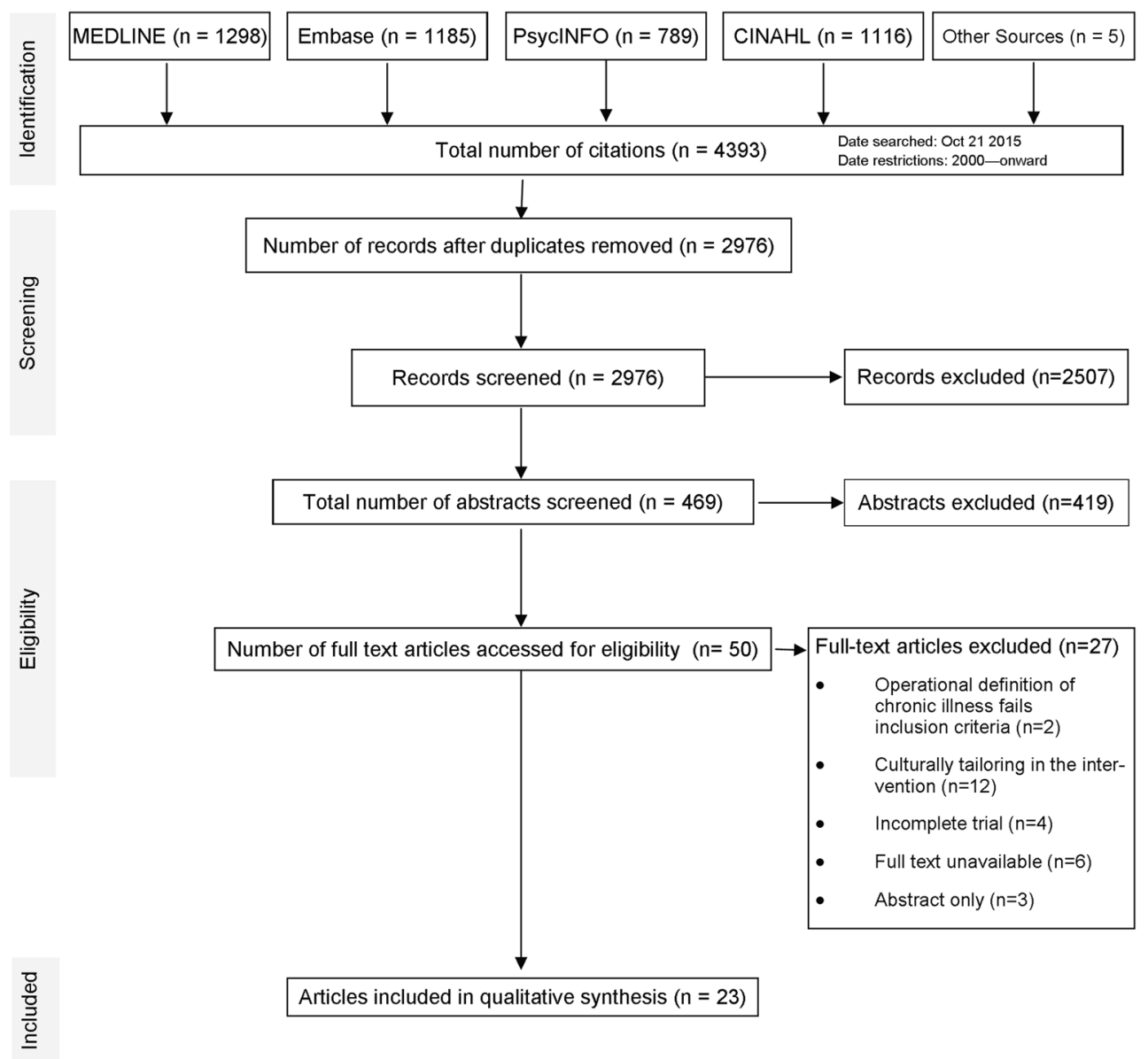

Fig. 1 PRISMA flow diagram. This figure illustrates the methodological flow of our study selection 
Table 1 Characteristics of Included Studies $(N=23)$

\begin{tabular}{|c|c|c|c|c|c|c|}
\hline $\begin{array}{l}\text { Author, Year, } \\
\text { Country }\end{array}$ & Goal & Population & Language & $\begin{array}{c}\text { Intervention (I) } \\
\text { Control (C) }\end{array}$ & $\begin{array}{c}\text { Outcomes* } \\
\text { Primary (P) } \\
\text { Secondary (S) }\end{array}$ & $\begin{array}{c}\text { Quality } \\
\text { summary } \\
\text { score (/11) }\end{array}$ \\
\hline \multicolumn{7}{|c|}{ Low Income } \\
\hline \multicolumn{7}{|c|}{ Diabetes } \\
\hline $\begin{array}{l}\text { Arora et al. } \\
(\underline{35)} \\
\text { USA }\end{array}$ & $\begin{array}{l}\text { Evaluate a text } \\
\text { message-based } \\
\text { mobile health } \\
\text { intervention (TExT- } \\
\text { MED) }\end{array}$ & $\begin{array}{l}\text { Sample size: } 128 \\
\text { Mean Age: } \\
\text { C: } 51.0 \text {, I: } 50.5 \\
\% \text { Male: } \\
\text { C: } 31 \%, \text { I: } 40 \% \\
\text { Ethnicity: } \\
\text { C: } 89 \% \text {; I: } 86 \% \\
\text { Latino } \\
\text { Income Measure: } \\
\text { Safety-net hospital }\end{array}$ & $\begin{array}{l}\text { English or } \\
\text { Spanish }\end{array}$ & $\begin{array}{l}\text { I: TExT-MED text messages to } \\
\text { enhance self-care behaviors } \\
\text { C: Usual Care } \\
\text { Duration of I: } 2 \text { text messages/day } \\
\text { x } 6 \text { month } \\
\text { Timing of measurements: } \\
\text { Baseline; } 6 \text { months }\end{array}$ & $\begin{array}{l}\text { P: No significant decrease in HbAlc levels } \\
\text { (data missing for Cohen's d) } \\
\text { S: Significant increase medication } \\
\text { adherence (Morisky Medication Adherence } \\
\text { Scale, d=0.11) } \\
\text { No significant increase in self-efficacy } \\
\text { (Diabetes Empowerment Scale SF, } \\
\text { d=0.12), QoL (Problem Areas in Diabetes } \\
\text { Scale, d=-0.39 @ } 6 \text { months), diabetes } \\
\text { knowledge (Michigan Diabetes knowledge } \\
\text { Test, d=0.04), and performance of self-care } \\
\text { tasks (Summary of Diabetes Self Care } \\
\text { Activities Scale) on subscales of general } \\
\text { diet (d=0.09), specific diet (d=0.18), } \\
\text { exercise (d=0.04), blood glucose testing } \\
\text { (d=0.04) and foot care d= - } 0.04 \text { )). No } \\
\text { significant decrease in ED utilization (data } \\
\text { missing to calculate Cohen's d) }\end{array}$ & 7 \\
\hline $\begin{array}{l}\text { Fischer et al. } \\
(\underline{36}) \\
\text { USA }\end{array}$ & $\begin{array}{l}\text { Evaluate different } \\
\text { modes of delivering } \\
\text { a patient Report } \\
\text { Card (PRC) }\end{array}$ & $\begin{array}{l}\text { Sample size: } 5457 \\
\text { Mean Age: } \\
\text { C+I: } 54.1 \\
\text { \% Male: } \\
\text { C+I: } 40.7 \\
\text { Ethnicity: } \\
\text { C+I: } 61.5 \% \text { Latino } \\
\text { Income Measure: } \\
\text { Federally-qualified } \\
\text { community health } \\
\text { centers, within an } \\
\text { urban safety-net } \\
\text { healthcare system }\end{array}$ & $\begin{array}{l}\text { English or } \\
\text { Spanish }\end{array}$ & $\begin{array}{l}\text { I: One of the following: (1) Four, } \\
\text { quarterly, mailed PRC, (2) } \\
\text { Distribution of automatically } \\
\text { generated PRC at the point-of-care, } \\
\text { or (3) HCP PRC (standard or } \\
\text { enhanced) generated from the } \\
\text { diabetes registry and distributed to } \\
\text { providers as a quarterly email } \\
\text { C: Usual care } \\
\text { Duration of I: Received PRCs } \\
\text { every } 3 \text { months x } 12 \text { months; met } \\
\text { with HCP as needed for } 13 \text { months } \\
\text { Timing: Baseline, } 13 \text { months }\end{array}$ & $\begin{array}{l}\text { Mailed PRC = No significant difference } \\
\text { across glycemic control, lipids, and BP } \\
\text { (data missing for Cohen's d) } \\
\text { Point-of-care PRC = No significant } \\
\text { difference lipid target, usual care } \\
\text { significantly better glycemic control and } \\
\text { BP (data missing for Cohen's d) } \\
\text { Enhanced provider PRC = Significant } \\
\text { better glycemic control, no significant } \\
\text { differences for lipid and BP (data missing } \\
\text { for Cohen's d) } \\
\text { Point-of-care PRC + Enhanced } \\
\text { provider PRC = Significantly worse BP } \\
\text { control, no significant outcomes for } \\
\text { glycemic control and lipid (data missing } \\
\text { for Cohen's d) }\end{array}$ & 7 \\
\hline $\begin{array}{l}\text { Fischer et al. } \\
(\underline{37)} \\
\text { USA }\end{array}$ & $\begin{array}{l}\text { Evaluate the } \\
\text { effectiveness of a } \\
\text { nurse-led telephone } \\
\text { care intervention } \\
\text { (SM techniques, } \\
\text { motivational } \\
\text { interviewing) on } \\
\text { improving lipid } \\
\text { control in adult } \\
\text { patients with Type II } \\
\text { diabetes }\end{array}$ & $\begin{array}{l}\text { Sample size: } 762 \\
\text { Mean Age: } \\
\text { C: } 58.3 \text {, I: } 58.5 \\
\text { \% Male: } \\
\text { C: } 42.8 \%, \text { I: } 36.0 \% \\
\text { Ethnicity: } \\
\text { C: } 81.1 \% ; \text { I: } 81.6 \% \\
\text { Latino } \\
\text { Income Measure: } \\
\text { Federally-funded } \\
\text { community health } \\
\text { centers }\end{array}$ & $\begin{array}{l}\text { English or } \\
\text { Spanish }\end{array}$ & $\begin{array}{l}\text { I: Nurse-led telephone outreach } \\
\text { program focused on lipid } \\
\text { management. Adjustment of LDL } \\
\text { medications by nurses based on lab } \\
\text { results + on-going medication } \\
\text { monitoring + motivational } \\
\text { interviewing and facilitated patient } \\
\text { SM } \\
\text { C: Usual care } \\
\text { Duration of I: } 20 \text { months } \\
\text { Timing of measurements: } \\
\text { Baseline, } 20 \text { months }\end{array}$ & $\begin{array}{l}\text { P: Significantly fewer patients LDL }<100 \\
\mathrm{mg} / \mathrm{dL} \text { (data missing for Cohen's d) } \\
\text { S: No significant difference in outpatient } \\
\text { and ED visits and BP (data missing for } \\
\text { Cohen's d) } \\
\text { Significant decrease in average cost per } \\
\text { patient to the health care system, } \\
\text { significantly more patients with CVD } \\
\text { with an LDL less than } 70 \mathrm{mg} / \mathrm{dL} \text { (data } \\
\text { missing for Cohen's d) }\end{array}$ & 5 \\
\hline $\begin{array}{l}\text { Frosch et al. } \\
(\underline{38}) \\
\text { USA }\end{array}$ & $\begin{array}{l}\text { Evaluate the } \\
\text { effectiveness of a } \\
\text { behavior support } \\
\text { intervention (video, } \\
\text { workbook, } \\
\text { telephone coaching } \\
\text { with diabetes } \\
\text { specialist nurse) on } \\
\text { decreasing HbA1c } \\
\text { levels in patients } \\
\text { with poorly } \\
\text { controlled diabetes }\end{array}$ & $\begin{array}{l}\text { Sample size: } 201 \\
\text { Mean Age: } \\
\text { C: } 54.3 \text {, I: } 56.7 \\
\% \text { Male: } \\
\text { C: } 57 \% \text {, I: } 46 \% \\
\text { Ethnicity: } \\
\text { C: } 71 \% \text {; I: } 72.8 \% \\
\text { African American or } \\
\text { Latino } \\
\text { Income measure: } \\
74 \% \text { incomes of } \leq \\
\$ 15000\end{array}$ & $\begin{array}{l}\text { English or } \\
\text { Spanish }\end{array}$ & $\begin{array}{l}\text { I: DVD program + workbook } \\
\text { "Living with Diabetes: Making } \\
\text { Lifestyle Changes to Last a } \\
\text { Lifetime" + maximum of } 5 \\
\text { telephone coaching sessions with a } \\
\text { bilingual nurse educator. } \\
\text { C: Usual care; patients given } \\
\text { booklet " } 4 \text { Steps to Control Your } \\
\text { Diabetes for Life" } \\
\text { Duration of I: } 24 \text { minute video + } \\
\text { up to } 5 \text { sessions of telephone } \\
\text { coaching ( } 1 \text { x } 60 \text { min.; } 2 \text { x } 30 \text { min; } \\
\text { and } 2 \text { x } 15 \text { min) } \\
\text { Timing of measurements: }\end{array}$ & $\begin{array}{l}\text { P: No significant reduction in mean } \\
\text { HbAlc }(\mathrm{d}=-0.16) \\
\text { S: Significant improvement self-care } \\
\text { behaviors (Summary of Diabetes Self } \\
\text { Care Activities Scale) exercise ( } \mathrm{d}=-0.16) \\
\text { No significant changes in LDL }(\mathrm{d}=0.22) \text {, } \\
\text { systolic BP }(\mathrm{d}=0.05) \text {, diastolic BP } \\
(\mathrm{d}=0.07), \mathrm{BMI}(\mathrm{d}=0.07) \text {, diabetes } \\
\text { knowledge (Michigan Diabetes } \\
\text { knowledge Test, } \mathrm{d}=0.05) \text {, and self-care } \\
\text { behaviors (Summary of Diabetes Self } \\
\text { Care Activities Scale) of general diet }(\mathrm{d}=- \\
0.02) \text {, specific diet }(\mathrm{d}=0.06) \text {, blood } \\
\text { glucose testing }(\mathrm{d}=-0.10) \text {, foot care }\end{array}$ & 9 \\
\hline
\end{tabular}




\section{Table 1 (continued)}

\begin{tabular}{|c|c|c|c|c|c|c|}
\hline & & & & Baseline, 1 month and 6 months & $\begin{array}{l}(\mathrm{d}=0.02), \text { taking most of } \mathrm{Rx} \text { medications } \\
(\mathrm{d}=-0.19) \text {, and taking all of } \mathrm{Rx} \\
\text { medications }(\mathrm{d}=-0.34)\end{array}$ & \\
\hline $\begin{array}{l}\text { Gregg et al. } \\
(\underline{39)} \\
\text { USA }\end{array}$ & $\begin{array}{l}\text { Enhance coping } \\
\text { with diabetes using } \\
\text { an acceptance and } \\
\text { mindfulness } \\
\text { intervention } \\
\text { amongst adults with } \\
\text { Type II diabetes }\end{array}$ & $\begin{array}{l}\text { Sample size: } 81 \\
\text { Mean Age: } \\
\text { C: } 49.8 \text {, I: } 51.9 \\
\text { \% Male: } \\
\text { C: } 42.1 \% \text {, I: } 51.2 \% \\
\text { Ethnicity: } \\
\text { C: } 26.2 \% ; \text { I: } 30.2 \% \\
\text { Latino } \\
\text { Income measure: } \\
\text { "Low-income } \\
\text { community health } \\
\text { center" }\end{array}$ & English & $\begin{array}{l}\text { I: Researcher-led 4-hour education } \\
\text { workshop + acceptance and } \\
\text { Commitment Therapy (ACT) } \\
\text { session } \\
\text { C: Education alone attention } \\
\text { control including a 7-hour } \\
\text { workshop } \\
\text { Duration of I: One 4-hour } \\
\text { education workshop + ACT } \\
\text { sessions (time not specified) } \\
\text { Timing of measurements: } \\
\text { Baseline; } 3 \text { months }\end{array}$ & $\begin{array}{l}\text { P: Significant increase in diabetic control } \\
\text { in intervention group compared to control } \\
\text { (HbA1c levels }<7.0 \% \text {, data missing to } \\
\text { calculate Cohen's d) } \\
\text { S: Significant increase in diabetes } \\
\text { acceptance (Acceptance and Action } \\
\text { Diabetes Questionnaire, d=0.38) and SM } \\
\text { behavior (Diabetes Adherence Measure, } \\
\text { exercise, diet and glucose monitoring, } \\
\text { d=0.2) } \\
\text { No significant increase in diabetes } \\
\text { knowledge (Diabetes care profile, } d=0.5 \text { ), } \\
\text { or absolute HbA1c levels (d=-0.3) }\end{array}$ & 9 \\
\hline $\begin{array}{l}\text { Piette et al. } \\
\text { (40) } \\
\text { USA }\end{array}$ & $\begin{array}{l}\text { Evaluate the } \\
\text { effectiveness of } \\
\text { automated telephone } \\
\text { disease management } \\
\text { (ATDM) } \\
\text { intervention to } \\
\text { improve diabetes } \\
\text { SM and QoL } \\
\text { measures in adults } \\
\text { with diabetes }\end{array}$ & $\begin{array}{l}\text { Sample size: } 248 \\
\text { Mean Age: } \\
\text { C: } 53.3 \text {, I: } 55.7 \\
\text { \% Male: } \\
\text { C: } 43.5 \%, \text { I: } 38.7 \% \\
\text { Ethnicity: } \\
\text { C: } 51.6 \% \text {; I: } 47.6 \% \\
\text { Latino } \\
\text { Income Measure: } \\
\text { C: } 56.5 \% \text {; I: } 59.1 \% \\
\text { < } 10,000 \text { per annum }\end{array}$ & $\begin{array}{l}\text { English or } \\
\text { Spanish }\end{array}$ & $\begin{array}{l}\text { I: Standardized, computer- } \\
\text { generated, weekly, 5-8 min } \\
\text { telephone assessments on glycemic } \\
\text { self-monitoring and diabetes self- } \\
\text { care + option to receive } 30-60 \\
\text { second health tips + option of } 3-7 \\
\text { minutes interactive dietary } \\
\text { education module + option to } \\
\text { receive nurse-led follow up calls as } \\
\text { needed. } \\
\text { C: Usual care } \\
\text { Duration of I: Bi-weekly calls for } \\
12 \text { months } \\
\text { Timing of measurements: } \\
\text { Baseline; } 12 \text { months }\end{array}$ & 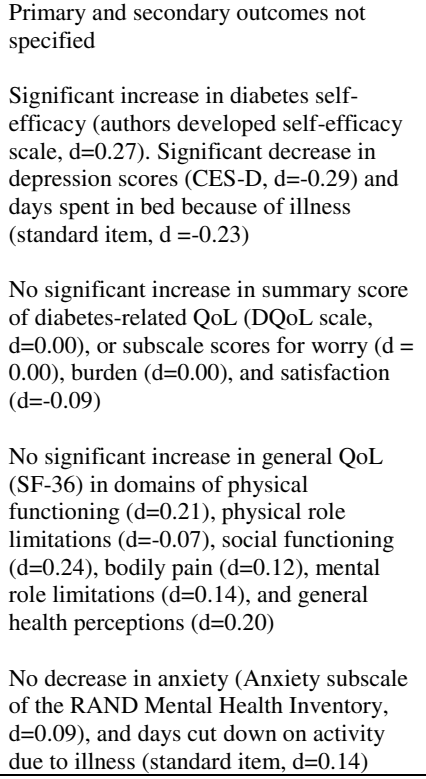 & 9 \\
\hline $\begin{array}{l}\text { Sequeira et al. } \\
(\underline{41)} \\
\text { USA }\end{array}$ & $\begin{array}{l}\text { Develop and } \\
\text { implement } \\
\text { continuous glucose } \\
\text { monitoring (CGM) } \\
\text { in an existing } \\
\text { diabetes clinic for } \\
\text { low-income Type } 1 \\
\text { diabetes patients }\end{array}$ & $\begin{array}{l}\text { Sample size: } 39 \\
\text { Mean Age: } \\
\text { C+I: } 40 \\
\text { \% Male: } \\
\text { C+I: } 52 \\
\text { Ethnicity: } \\
\text { C+I: } 76 \% \text { Latino } \\
\text { Income measure: } \\
\text { clinic for low-income } \\
\text { patients }\end{array}$ & $\begin{array}{l}\text { English or } \\
\text { Spanish }\end{array}$ & $\begin{array}{l}\text { I: Physician-led continuous } \\
\text { glucose monitoring program } \\
\text { C: SMBG (cross-over design) } \\
\text { Duration of I: monthly visits over } \\
21 \text { weeks } \\
\text { Timing of measurements: } \\
\text { Baseline; monthly visit }\end{array}$ & $\begin{array}{l}\text { Primary and secondary outcomes not } \\
\text { specified } \\
\text { No significant difference in HbA1c levels } \\
\text { (data missing to calculate Cohen's d) or } \\
\text { time in parameters of low or high blood } \\
\text { glucose (data missing to calculate } \\
\text { Cohen's d) }\end{array}$ & 3 \\
\hline $\begin{array}{l}\text { Thom et al. } \\
\text { (42) } \\
\text { USA }\end{array}$ & $\begin{array}{l}\text { Evaluate the impact } \\
\text { of a peer coaching } \\
\text { intervention on } \\
\text { glucose control on } \\
\text { patients with poorly } \\
\text { controlled diabetes } \\
\text { attending public } \\
\text { clinics }\end{array}$ & $\begin{array}{l}\text { Sample size: } 299 \\
\text { Mean Age: } \\
\text { C: } 54.1 \text {, I: } 56.3 \\
\text { \% Male: } \\
\text { C: } 47 \% \text { I: } 48.6 \% \\
\text { Ethnicity: } \\
\text { C: } 48.7 \% ; \text { I: } 44.6 \% \\
\text { White Hispanic } \\
\text { Income Measure: } \\
\text { Public health clinic } \\
\text { C: } 60.4 \% ; \text { I: } 60.8 \%< \\
\text { \$10,000 per annum }\end{array}$ & $\begin{array}{l}\text { English or } \\
\text { Spanish }\end{array}$ & $\begin{array}{l}\text { I: Peer coach-led sessions (face-to- } \\
\text { face \& telephone sessions) for } \\
\text { action planning } \\
\text { C: Usual care including access to a } \\
\text { nutritionist and diabetes educator } \\
\text { Duration of I: Two face-to-face } \\
\text { contacts \& two telephone contacts } \\
\text { per month for } 6 \text { months } \\
\text { Timing of measurements: } \\
\text { Baseline, and } 6 \text { months }\end{array}$ & $\begin{array}{l}\text { P: Significant decrease in HbA1c level } \\
(\mathrm{d}=-0.27) \\
\text { S: Significantly more participants had a } \\
\text { drop of } 1.0 \% \text { in HbAlc }(\mathrm{OR}=2.14) \\
\\
\text { No significant decrease in proportion of } \\
\text { patients with HbA1c level of less than } \\
7.5 \%(\mathrm{OR}=1.61) \text {. No significant decrease } \\
\text { in LDL levels }(\mathrm{d}=-0.08) \text {, systolic BP } \\
(\mathrm{d}=0.2) \text {, and BMI }(\mathrm{d}=0.26)\end{array}$ & 10 \\
\hline \multicolumn{7}{|c|}{ Hypertension } \\
\hline $\begin{array}{l}\text { Bove et al. } \\
(\underline{43)} \\
\text { USA }\end{array}$ & $\begin{array}{l}\text { Evaluate the } \\
\text { effectiveness of a } \\
\text { telephone and } \\
\text { Internet-based } \\
\text { system for }\end{array}$ & $\begin{array}{l}\text { Sample size: } 241 \\
\text { Mean Age: } \\
\text { C: } 58.2, \text { I: } 61.0 \\
\text { \% Male: } \\
\text { C: } 35 \%, \text { I: } 35 \%\end{array}$ & $\begin{array}{l}\text { Not } \\
\text { specified } \\
\text { (implicit } \\
\text { English } \\
\text { only) }\end{array}$ & $\begin{array}{l}\text { I: Computer-generated email or } \\
\text { telephone reminder to report health } \\
\text { data Web form or through the } \\
\text { telephone; Nurse-led follow up } \\
\text { telephone or internet response for }\end{array}$ & $\begin{array}{l}\text { P: No significant decrease in BP to } \\
\text { systolic target of }<140 \mathrm{mmHg} \text { (data } \\
\text { missing to calculate Cohen's D). No } \\
\text { significant decrease in absolute systolic } \\
\text { BP (d=-0.13), or diastolic BP (d=-0.08) }\end{array}$ & 6 \\
\hline
\end{tabular}




\section{Table 1 (continued)}

\begin{tabular}{|c|c|c|c|c|c|c|}
\hline & $\begin{array}{l}\text { delivering a patient- } \\
\text { centered self- } \\
\text { monitoring program } \\
\text { for hypertension } \\
\text { management in an } \\
\text { underserved, urban } \\
\text { community with a } \\
\text { high incidence of } \\
\text { hypertension and } \\
\text { diabetes }\end{array}$ & $\begin{array}{l}\text { Ethnicity: } \\
\text { C: } 81 \% ; \text { I: } 80 \% \\
\text { African American } \\
\text { Income Measure: } \\
\text { clinic in underserved } \\
\text { area (50\% had family } \\
\text { incomes at or near } \\
\text { the poverty level) }\end{array}$ & & $\begin{array}{l}\text { patients with elevated BP } \\
\text { C: Usual care + provided data } \\
\text { from baseline assessment and } \\
\text { advised to contact primary care } \\
\text { provider for further care } \\
\text { Duration of I: } 2 \text { contacts per week } \\
\text { for } 6 \text { months } \\
\text { Timing of measurements: } \\
\text { Baseline and } 6 \text { months }\end{array}$ & $\begin{array}{l}\text { S: No significant changes in fasting blood } \\
\text { glucose }(\mathrm{d}=-0.08) \text {, total cholesterol }(\mathrm{d}=- \\
0.23), \mathrm{HDL} \text { levels }(\mathrm{d}=0.03), \text { LDL levels } \\
(\mathrm{d}=-0.12) \text {, triglycerides }(\mathrm{d}=-0.18) \text {, or BMI } \\
(\mathrm{d}=-0.13) \text { between groups in exploratory } \\
\text { analyses }\end{array}$ & \\
\hline $\begin{array}{l}\text { Martin et al. } \\
(\underline{44}) \\
\text { USA }\end{array}$ & $\begin{array}{l}\text { Determine the } \\
\text { effectiveness of a } \\
\text { community-based, } \\
\text { multimedia } \\
\text { intervention on } \\
\text { medication } \\
\text { adherence among } \\
\text { hypertensive adults }\end{array}$ & $\begin{array}{l}\text { Sample size: } 434 \\
\text { Mean Age: } \\
\text { C: } 55.2 \text {, I: } 56.9 \\
\text { \% Male: } \\
\text { C: } 30.5 \% \text {, I: } 34.4 \% \\
\text { Ethnicity: } \\
\text { C: } 94.8 \% ; \text { I: } 94.6 \% \\
\text { African American } \\
\text { Income Measure: } \\
\text { Federally Qualified } \\
\text { Health Center } \\
\text { C: } 82.2 \% ; \text { I: } 81.4 \%< \\
\text { \$15, } 000 \text { per annum }\end{array}$ & $\begin{array}{l}\text { Not } \\
\text { specified } \\
\text { (implicit } \\
\text { English } \\
\text { only) }\end{array}$ & $\begin{array}{l}\text { I: Delivered via a "Cyber Nurse" } \\
\text { and a "Video Doc" with a } \\
\text { community health advisor (CHA). } \\
\text { The CHA conducted } 4 \text { home visits, } \\
\text { each visit followed by a phone call } \\
\text { C: } 4 \text { home visits by CHA to } \\
\text { deliver computer-based video } \\
\text { education } \\
\text { Duration of I: } 4 \text { sessions over } 6 \\
\text { months } \\
\text { Timing of measurements: } \\
\text { Baseline, } 12 \text { months }\end{array}$ & $\begin{array}{l}\text { P: No significant increase in medication } \\
\text { adherence }(\mathrm{OR}=0.95) \\
\begin{array}{l}\text { S: No significant change in depression } \\
\text { (Center for Epidemiologic Studies }\end{array} \\
\begin{array}{l}\text { Depression scale, insufficient data to } \\
\text { calculate Cohen's D) }\end{array}\end{array}$ & 5 \\
\hline \multicolumn{7}{|c|}{ Obesity } \\
\hline $\begin{array}{l}\text { Bennett et al. } \\
(\underline{45)} \\
\text { USA }\end{array}$ & $\begin{array}{l}\text { Evaluate the } \\
\text { effectiveness of a } \\
\text { behavioral } \\
\text { intervention focused } \\
\text { on weight loss and } \\
\text { adherence to } \\
\text { individual HTN } \\
\text { medication regiment } \\
\text { for primary care } \\
\text { patients }\end{array}$ & $\begin{array}{l}\text { Sample size: } 365 \\
\text { Mean Age: } \\
\text { C: } 54.7, \text { I: } 54.6 \\
\text { \% Male: } \\
\text { C: } 34.1 \%, \text { I: } 28.9 \% \\
\text { Ethnicity: } \\
\text { C: } 70.8 \% ; \text { I: } 71.7 \% \\
\text { African American } \\
\text { Income Measure: } \\
\text { Urban community } \\
\text { health center that } \\
\text { served a } \\
\text { predominately } \\
\text { racial/ethnic } \\
\text { minority patient } \\
\text { population }\end{array}$ & $\begin{array}{l}\text { English or } \\
\text { Spanish }\end{array}$ & $\begin{array}{l}\text { I: Community health-educator led } \\
\text { session involving } 3 \text { tailored goals } \\
\text { to modify health behaviors; self- } \\
\text { monitoring via website or } \\
\text { telephone; telephone-based follow- } \\
\text { up and provision of community } \\
\text { resources } \\
\text { C: Usual care + self-help booklet } \\
\text { Duration of I: } 1 \text { initial session and } \\
\text { goal revision every } 13 \text { weeks; } \\
\text { Monthly telephone-based } \\
\text { counseling x } 12 \text { months followed } \\
\text { by bi-monthly counseling x next } \\
12 \text { months } \\
\text { Timing of measurements: } \\
\text { Baseline, } 6-, 12-, 18 \text { - and } 24- \\
\text { months }\end{array}$ & $\begin{array}{l}\text { P: Significant decrease in body weight (d } \\
=-0.09) \\
\text { S: No significant decrease in systolic BP } \\
(d=-0.10)\end{array}$ & 9 \\
\hline \multicolumn{7}{|c|}{ Asthma } \\
\hline $\begin{array}{l}\text { Krieger et al. } \\
(\underline{46}) \\
\text { USA }\end{array}$ & $\begin{array}{l}\text { Evaluate the impact } \\
\text { of home visits by a } \\
\text { Community Health } \\
\text { Worker (CHW) on } \\
\text { QoL for adults with } \\
\text { asthma }\end{array}$ & $\begin{array}{l}\text { Sample size: } 366 \\
\text { Mean Age: } \\
\text { C: } 41.3 \text {, I: } 41.2 \\
\text { \% Male: } \\
\text { C: } 27 \%, \text { I: } 26.6 \\
\text { Ethnicity: } \\
\text { C: } 45 \% ; \text { I: } 48.6 \% \\
\text { Latino } \\
\text { Income Measure: } \\
\text { Household income } \\
<250 \% \text { of } 2007 \\
\text { Federal poverty line }\end{array}$ & $\begin{array}{l}\text { English or } \\
\text { Spanish }\end{array}$ & $\begin{array}{l}\text { I: CHW led home visits to provide } \\
\text { education, support, and service } \\
\text { coordination tailored to current } \\
\text { knowledge and asthma status + } \\
\text { four follow-up contacts } \\
\text { C: Usual care + education } \\
\text { pamphlets and information about } \\
\text { community resources } \\
\text { Duration of I: } 5 \text { visits (baseline, } \\
0.5,1.5,3.5 \text { and } 7 \text { months) and } \\
\text { follow up phone/email support as } \\
\text { needed } \\
\text { Timing of measurements: } 12 \\
\text { months }\end{array}$ & $\begin{array}{l}\text { P: Significant increase in asthma } \\
\text { symptom free-days }(\mathrm{d}=0.56) \text { and } \\
\text { improved asthma QoL (Mini Asthma } \\
\text { QoL Questionnaire, } d=0.81) \\
\text { No significant difference in urgent care } \\
\text { episodes between groups }(\mathrm{d}=-0.08) \\
\text { S: Significant improvements in night } \\
\text { symptoms }(\mathrm{d}=-0.32) \text {, asthma control }(\mathrm{d}= \\
-0.70) \text {, and general physical health status } \\
(\text { SF-12, } \mathrm{d}=1.06) \text { and decreased asthma } \\
\text { exacerbation }(\mathrm{d}=-0.32) \text { and day use of } \\
\text { rescue medication }(\mathrm{d}=0.42) \\
\\
\text { No significant improvements in } \\
\text { pulmonary function }(\mathrm{d}=0.00) \text {, use of oral } \\
\text { steroid burst }(\mathrm{d}=-0.05), \text { days missed at } \\
\text { work and/or school }(\mathrm{d}=-0.07) \text {, or general } \\
\text { mental health status }(\mathrm{SF}-12, \mathrm{~d}=0.05)\end{array}$ & 10 \\
\hline \multicolumn{7}{|c|}{ Multiple Chronic Illnesses } \\
\hline $\begin{array}{l}\text { Riley et al. } \\
(\underline{47)} \\
\text { USA }\end{array}$ & $\begin{array}{l}\text { Evaluate the } \\
\text { effectiveness of } \\
\text { tailored counseling } \\
\text { in increasing use of } \\
\text { social- } \\
\text { environmental } \\
\text { resources to support } \\
\text { SM of chronic } \\
\text { conditions }\end{array}$ & $\begin{array}{l}\text { Sample size: } 28 \\
\text { Mean Age: } \\
\text { C+I: } 58 \\
\text { \% Male: } \\
\text { C: } 8 \% \text {, I: } 27 \% \\
\text { Ethnicity: } \\
\text { C: } 67 \% \text {;: } 47 \% \\
\text { Caucasian } \\
\text { Income Measure: } \\
\text { Low income } \\
\text { community health } \\
\text { center }\end{array}$ & English & $\begin{array}{l}\text { I: Health educator led visit } \\
\text { addressing use of social- } \\
\text { environmental resources, specific } \\
\text { SM goal-planning, barriers, and } \\
\text { action-plan; } 1 \text { follow up call and } 2 \\
\text { newsletters } \\
\text { C: Wait-list control } \\
\text { Duration of I: 1-hour session, } 5 \\
\text { minute follow up call one week } \\
\text { later, a generic newsletter } \\
\text { delivered after visit, and a tailored }\end{array}$ & $\begin{array}{l}\text { P: Significant increase in use of social- } \\
\text { environmental resources (Chronic Illness } \\
\text { Resources Survey, sample size too small } \\
\text { to calculate Cohen's D) } \\
\text { S: Significant increase in medication } \\
\text { adherence (Medical Outcomes Study } \\
\text { Illness Management scale, sample size } \\
\text { too small to calculate Cohen's D) and } \\
\text { minutes of physical activity per week } \\
\text { (Behavioral Risk Factor Surveillance } \\
\text { System, sample size too small to calculate }\end{array}$ & 3 \\
\hline
\end{tabular}


Table 1 (continued)

\begin{tabular}{|c|c|c|c|c|c|c|}
\hline & & & & $\begin{array}{l}\text { newsletter delivered } 5 \text { weeks later } \\
\text { Timing of measurements: } \\
\text { Baseline and } 1 \text { and } 3 \text { months } \\
\text { follow-ups }\end{array}$ & $\begin{array}{l}\text { Cohen's D) } \\
\text { No significant improvements in minutes } \\
\text { of walking (Behavioral Risk Factor } \\
\text { Surveillance System) and eating patterns } \\
\text { (Kristal Fat and Fiber Behavior scale). }\end{array}$ & \\
\hline \multicolumn{7}{|c|}{ Low Health Literacy } \\
\hline \multicolumn{7}{|c|}{ Arthritis } \\
\hline $\begin{array}{l}\text { Rudd et al. } \\
(\underline{48)} \\
\text { USA }\end{array}$ & $\begin{array}{l}\text { Evaluate the } \\
\text { efficacy of plain } \\
\text { language } \\
\text { information } \\
\text { materials and/or } \\
\text { individualized } \\
\text { sessions to reduce } \\
\text { literacy barriers and } \\
\text { enhance health } \\
\text { outcomes among } \\
\text { patients with } \\
\text { inflammatory } \\
\text { arthritis. }\end{array}$ & $\begin{array}{l}\text { Sample size: } 127 \\
\text { Mean age: } \\
\text { C: } 59.5, \mathrm{I}_{1+2}: 57.6 \\
\text { \% Male: } \\
\text { C: } 22 \%, \mathrm{I}_{1+2}: 19 \% \\
\text { Ethnicity: } \\
\text { C: } 94 \% ; \mathrm{I}_{1+2}: 91 \% \\
\text { Caucasian } \\
\text { Literacy Measure: } \\
\text { A-REALM } \\
\text { C: } 21 \% ; \mathrm{I}_{1+2}: 16 \% \\
\text { had high school or } \\
\text { less }\end{array}$ & $\begin{array}{l}\text { Not } \\
\text { specified } \\
\text { implicit } \\
\text { English } \\
\text { only }\end{array}$ & $\begin{array}{l}\mathbf{I}_{1} \text { Plain language information } \\
\text { materials: educational notebook } \\
\text { written at a } 5^{\text {th }}-8^{\text {th }} \text { grade reading } \\
\text { level } \\
\mathbf{I}_{2} \text { Individualized Care Group: } \\
\text { educational notebook + two } \\
\text { individual 1-hour meetings with } \\
\text { the educator addressing self-care, } \\
\text { barriers and communication } \\
\text { strategies } \\
\text { C: Usual care; Arthritis } \\
\text { Foundation pamphlets ( } 11^{\text {th }}-15^{\text {th }} \\
\text { reading level) } \\
\text { Duration of } \mathbf{I}_{2} \text { : Two 1-hour } \\
\text { sessions + additional support as } \\
\text { needed } \\
\text { Timing of measurements: } \\
\text { Baseline, } 6 \text { months, and } 12 \text { months }\end{array}$ & $\begin{array}{l}\text { P: Significant improvement in self- } \\
\text { efficacy (Lorig's self-efficacy scale, } d= \\
0.21 \text { ). } \\
\text { No significant improvements in } \\
\text { adherence to treatment (4-item measure } \\
\text { based on questionnaire by Levine, } d=- \\
\text { 0.04), satisfaction with care (Medical } \\
\text { Interview Satisfaction Scale, } d=-0.23 \text { ), } \\
\text { and appointment keeping (self-efficacy } \\
\text { scale, d=-0.17) } \\
\text { S: No significant improvements in mental } \\
\text { health scores (SF-36, d=0.32) or in health } \\
\text { status (Health Assessment Questionnaire, } \\
d=0.08 \text { ) }\end{array}$ & 6 \\
\hline \multicolumn{7}{|c|}{ Cardiovascular Diseases } \\
\hline $\begin{array}{l}\text { DeWalt et al. } \\
(\underline{49)} \\
\text { USA }\end{array}$ & $\begin{array}{l}\text { Compare the } \\
\text { efficacy of a } \\
\text { single session versus } \\
\text { a more intensive } \\
\text { multisession } \\
\text { education program } \\
\text { among patients with } \\
\text { heart failure. }\end{array}$ & $\begin{array}{l}\text { Sample size: } 605 \\
\text { Mean age: } \\
\text { C: } 60.3 \text {, I: } 61.1 \\
\text { \% Male: } \\
\text { C: } 52 \% \text {, I: } 52 \% \\
\text { Ethnicity: } \\
\text { C: } 40 \% \text { Caucasian; } \\
\text { 38\% African } \\
\text { American } \\
\text { I: } 37 \% \text { Caucasian; } \\
\text { 39\% African } \\
\text { American } \\
\text { Literacy Measure: } \\
\text { S-TOFHLA }\end{array}$ & $\begin{array}{l}\text { English or } \\
\text { Spanish }\end{array}$ & $\begin{array}{l}\text { I: } 1 \text { x } 40 \text {-minute health educator } \\
\text { led session }+10 \text { minutes } 5-8 \\
\text { follow up phone calls } \\
\text { C: } 1 \text { x } 40 \text {-minute health educator } \\
\text { led session + usual care session } \\
\text { and self-care sessions } \\
\text { Duration of I: } 40 \text {-minute session } \\
+ \text { mandatory } 4 \text { weeks follow-up } \\
\text { telephone calls (10 minutes each)+ } \\
\text { additional telephone calls every } 2 \\
\text { weeks as needed } \\
\text { Timing of measurements: } \\
\text { All-cause Hosp.: Baseline, } 6 \text {, and } \\
12 \text { months. } \\
\text { HFQOL: Baseline, } 1,6 \text {, and } 12 \\
\text { months }\end{array}$ & $\begin{array}{l}\text { P: No significant decrease in all-cause } \\
\text { hospitalization or death (IRR = 0.96) } \\
\text { S: Significant increase in heart failure } \\
\text { related QoL (Chronic Illness Care } \\
\text { Evaluation Heart Failure Symptom Scale, } \\
\text { data missing to calculate Cohen's D). } \\
\text { No significant decrease in heart failure } \\
\text { related hospitalization (data missing to } \\
\text { calculate Cohen's D). or emergency } \\
\text { department visits (data missing to } \\
\text { calculate Cohen's D) }\end{array}$ & 9 \\
\hline $\begin{array}{l}\text { DeWalt et al. } \\
(\underline{50)} \\
\text { USA }\end{array}$ & $\begin{array}{l}\text { Evaluate the } \\
\text { efficacy of a heart } \\
\text { failure SM program } \\
\text { designed for patients } \\
\text { with low literacy. }\end{array}$ & $\begin{array}{l}\text { Sample size: } 127 \\
\text { Mean age: } \\
\text { C: } 62, \text { I: } 63 \\
\% \text { Male: } \\
\text { C: } 41 \% \text {, I: } 58 \% \\
\text { Ethnicity: } \\
\text { C: } 55 \% \text {; I: } 54 \% \text {, } \\
\text { African American } \\
\text { Literacy Measure: } \\
\text { S-TOFHLA (41\% } \\
\text { literacy < } 4^{\text {th }} \text { grade) }\end{array}$ & English & $\begin{array}{l}\text { I: } 1 \text {-hour HCP led session on signs } \\
\text { and symptoms, daily weight } \\
\text { monitoring and diuretic self- } \\
\text { adjustment. Patients received an } \\
\text { educational booklet written at the } \\
6^{\text {th }} \text { grade reading level. Face-to- } \\
\text { face session followed by } 10 \text { phone } \\
\text { calls x } 5-15 \text { minutes each } \\
\text { C: Usual care including a general } \\
\text { heart failure education pamphlet } \\
\text { written at the } 7^{\text {th }} \text { grade level. } \\
\text { Duration of I: } 1 \text {-hour educational } \\
\text { session }+10 \text { follow up calls x 5-15 } \\
\text { minutes each } \\
\text { Timing of measurements: } \\
\text { Baseline, } 6 \text { - and } 12 \text { - months }\end{array}$ & $\begin{array}{l}\text { P: No significant improvements in all- } \\
\text { cause hospitalizations, death (IRR = } \\
\text { 0.69), and heart failure-related QoL } \\
\text { (Minnesota Living with Heart Failure } \\
\text { Questionnaire, data missing to calculate } \\
\text { Cohen's D) } \\
\text { S: Significant improvements in heart } \\
\text { failure related knowledge (scale } \\
\text { developed by authors, data missing to } \\
\text { calculate Cohen's D), self-efficacy (scale } \\
\text { developed by authors, data missing to } \\
\text { calculate Cohen's D) and self-care } \\
\text { behaviors (how often patients weighed } \\
\text { themselves, OR = 9.21) }\end{array}$ & 7 \\
\hline $\begin{array}{l}\text { Kripalani et al. } \\
(\underline{51)} \\
\text { USA }\end{array}$ & $\begin{array}{l}\text { Evaluate the effect } \\
\text { of illustrated } \\
\text { medication } \\
\text { schedules and refill } \\
\text { reminder postcards } \\
\text { on medication } \\
\text { adherence in adults } \\
\text { with coronary heart } \\
\text { disease }\end{array}$ & $\begin{array}{l}\text { Sample size: } 440 \\
\text { Mean age: } \\
\mathrm{C}+\mathrm{I}_{1-3}: 63.7 \\
\text { \% Male: } \\
\mathrm{C}+\mathrm{I}_{1-3}: 44.4 \\
\text { Ethnicity: } \\
\mathrm{C}+\mathrm{I}_{1-3}: 91 \% \text { African } \\
\text { American } \\
\text { Literacy Measure: } \\
\text { REALM } \\
\mathrm{C}+\mathrm{I}_{1-3}: 45.1 \% \text { had a } \\
\text { literacy level } \leq 6 \text { th } \\
\text { grade }\end{array}$ & English & $\begin{array}{l}\text { I: Pharmacist-led intervention; } \\
\text { patients received: }\left(\mathrm{I}_{1}\right) \text { Illustrated } \\
\text { medication schedule; }\left(\mathrm{I}_{2}\right) \text { Refill } \\
\text { reminder post card; or }\left(\mathrm{I}_{3}\right) \text { both } \mathrm{I}_{1} \\
\text { and } \mathrm{I}_{2} \text {. } \\
\text { C: Usual care } \\
\text { Duration of I: } 5 \text { minute } \\
\text { orientation to medication schedule } \\
\text { and post-cards every } 2 \text { weeks (for } \\
\text { those in group } 2 \text { and } 3 \text { only) } \\
\text { Timing of measurements: } \\
\text { Baseline, } 1 \text { year }\end{array}$ & $\begin{array}{l}\text { P: No significant increase in medication } \\
\text { refill adherence (cumulative medication } \\
\text { gap, data missing to calculate Cohen's d) }\end{array}$ & 11 \\
\hline
\end{tabular}




\section{Table 1 (continued)}

\begin{tabular}{|c|c|c|c|c|c|c|}
\hline \multicolumn{7}{|c|}{$C O P D$} \\
\hline $\begin{array}{l}\text { Kiser et al. } \\
(\underline{52}) \\
\text { USA }\end{array}$ & $\begin{array}{l}\text { Examine the effect } \\
\text { of a literacy- } \\
\text { sensitive SM } \\
\text { intervention on } \\
\text { inhaler technique } \\
\text { scores in COPD } \\
\text { patients }\end{array}$ & $\begin{array}{l}\text { Sample size: } 99 \\
\text { Mean age: } \\
\text { C: } 63 \text {, I: } 63 \\
\text { \% Male: } \\
\text { C: } 34 \% \text {, I: } 36 \% \\
\text { Ethnicity: } \\
\text { C: } 72 \% \text {; : } 69 \% \\
\text { Caucasian } \\
\text { Literacy Measure: } \\
\text { S-TOFHLA } \\
\text { C: } 33 \% \text { I: } 37 \% \text { low } \\
\text { health literacy }\end{array}$ & English & $\begin{array}{l}\text { I: } 1 \text { x one-on-one, researcher-led } \\
\text { education session with a literacy- } \\
\text { sensitive handout and teach-back } \\
\text { method for inhaler use } \\
\text { C: Usual care } \\
\text { Duration of I: } 1 \text { x } 15-30 \text { session } \\
\text { Timing of measurements: } 2-8 \\
\text { weeks (until participant } \\
\text { demonstrated teach-back of inhaler } \\
\text { use) }\end{array}$ & $\begin{array}{l}\text { P: Significant increase in dose inhaler } \\
\text { technique score (data missing to calculate } \\
\text { Cohen's D) }\end{array}$ & 6 \\
\hline \multicolumn{7}{|c|}{ Cancer } \\
\hline $\begin{array}{l}\text { Wilson et al. } \\
(\underline{53}) \\
\text { USA }\end{array}$ & $\begin{array}{l}\text { Evaluate the } \\
\text { differential impact } \\
\text { of an audio-visual } \\
\text { education program } \\
\text { alone versus adding } \\
\text { a behavioral } \\
\text { contract to the } \\
\text { education program } \\
\text { among men with } \\
\text { prostate cancer }\end{array}$ & $\begin{array}{l}\text { Sample size: } 70 \\
\text { Mean age: } \\
\mathrm{C}+\mathrm{I}_{1+2}: 67.4 \\
\% \text { Male: } \\
\mathrm{C}+\mathrm{I}_{1+2}: 100 \% \\
\text { Ethnicity: } \\
\mathrm{C}+\mathrm{I}_{1+2}: 60 \% \text { African } \\
\text { American } \\
\text { Literacy Measure: } \\
\text { REALM }\end{array}$ & English & $\begin{array}{l}\text { I: Nurse-led intervention in which } \\
\text { patient received either }\left(\mathrm{I}_{1}\right) \text { an } \\
\text { education program or }\left(\mathrm{I}_{2}\right) \\
\text { Education plus behavioral } \\
\text { contracting after viewing the } 1^{\text {st }} \\
\text { video } \\
\text { C: Usual Care } \\
\text { Duration of I: (1) } 1 \times 15 \text { min video } \\
\text { at pre-treatment, treatment, and } \\
\text { post-treatment, (2) Same as (1) + } \\
\text { weekly one-on-one session to } \\
\text { review contract } \\
\text { Timing of measurements: } \\
\text { Baseline, } 3 \text { weeks, } 3 \text { months and } 6 \\
\text { months }\end{array}$ & $\begin{array}{l}\text { Primary and secondary outcomes not } \\
\text { specified. } \\
\text { No significant difference in self-care } \\
\text { behaviors (Side-Effect Interview and } \\
\text { Mood's Self-Care Management } \\
\text { Techniques Checklist, data missing to } \\
\text { calculate Cohen's D) of men in managing } \\
\text { radiation side effects }\end{array}$ & 5 \\
\hline \multicolumn{7}{|c|}{ Diabetes } \\
\hline $\begin{array}{l}\text { Cavanaugh et } \\
\text { al. }(\underline{54}) \\
\text { USA }\end{array}$ & $\begin{array}{l}\text { Evaluate the impact } \\
\text { of addressing both } \\
\text { literacy and } \\
\text { numeracy as part of } \\
\text { an enhanced } \\
\text { multidisciplinary } \\
\text { diabetes care } \\
\text { program }\end{array}$ & $\begin{array}{l}\text { Sample size: } 198 \\
\text { Median age: } \\
\text { C: 53, I: 52 } \\
\text { \% Male: } \\
\text { C: } 35 \%, \text { I: } 37 \% \\
\text { Ethnicity: } \\
\text { C: } 44 \% \text {; I: 42\% } \\
\text { African American } \\
\text { Income measure: } \\
\text { C: } 45 \% \text {; I: 52\% had < } \\
\text { \$20,000/year } \\
\text { Literacy Measure: } \\
\text { REALM C: 35\%; I: } \\
\text { 39\% literacy < } 9^{\text {th }} \\
\text { grade }\end{array}$ & English & $\begin{array}{l}\text { I: Healthcare professional led } \\
\text { intervention; patients received the } \\
24 \text { modules of the Diabetes } \\
\text { Literacy \& Numeracy Education } \\
\text { Toolkit (DLNET) which focuses } \\
\text { on self-care, nutrition } \\
\text { management, and medication } \\
\text { administration. } \\
\text { C: Enhanced usual care involving } \\
\text { 1-6 contacts with diabetes educator } \\
\text { or NP over a period of } 3 \text { months + } \\
\text { educational materials } \\
\text { Duration of I: } 2-6 \text { sessions over a } \\
\text { 3-month period. } \\
\text { Timing of measurements: } \\
\text { Baseline, } 3 \text { months and } 6 \text { months } \\
\text { (primary); baseline and } 6 \text { months } \\
\text { (secondary) }\end{array}$ & $\begin{array}{l}\text { P: Significant improvements in HbA1c at } \\
3 \text { months }(\mathrm{d}=-0.42) . \\
\text { No significant improvements in HbAlc at } \\
6 \text { months }(\mathrm{d}=-0.07) \\
\text { S: Significant increase in self-efficacy } \\
\text { (Perceived Diabetes } \\
\text { Self-Management Scale, } \mathrm{d}=0.23 \text { ). No } \\
\text { significant differences found for SM } \\
\text { behaviors (Diabetes Self-Care Activities } \\
\text { scale, (data missing to calculate Cohen's } \\
\text { D) or satisfaction (Diabetes Treatment } \\
\text { Satisfaction Questionnaire, } d=0.09)\end{array}$ & 9 \\
\hline \multicolumn{7}{|c|}{ Low Income and Low Health Literacy } \\
\hline \multicolumn{7}{|c|}{ Cardiovascular Disease } \\
\hline $\begin{array}{l}\text { Cordasca et al. } \\
(\underline{55)} \\
\text { USA }\end{array}$ & $\begin{array}{l}\text { Evaluate the effect } \\
\text { of a low-literacy } \\
\text { medication } \\
\text { education tool on } \\
\text { medication } \\
\text { adherence and } \\
\text { knowledge among } \\
\text { cardiac patients. }\end{array}$ & $\begin{array}{l}\text { Sample size: } 210 \\
\text { Mean Age: } \\
\text { C: } 55.7, \text { I: } 55.7 \\
\text { \% Male: } \\
\text { C: } 68.7 \% \text {, I: } 63.6 \% \\
\text { Ethnicity: } \\
\text { C: } 70 \% \text {; I: } 81 \% \\
\text { Latino } \\
\text { Income Measure: } \\
\text { Safety net hospital } \\
\text { LHL Measure: } \\
\text { TOFHLA C: } 43.4 \% \text {; } \\
\text { I: } 51.6 \% \text { had } \\
\text { inadequate literacy }\end{array}$ & \begin{tabular}{|l} 
English or \\
Spanish
\end{tabular} & $\begin{array}{l}\text { I: Nurse-led intervention in which } \\
\text { patient received a 30-day supply of } \\
\text { medications + teaching with low } \\
\text { literacy materials including a } \\
\text { customized medication tool } \\
\text { C: Usual care. } \\
\text { Duration of I: One session } \\
\text { Timing of measurements: Week } \\
2 \text { (12-18 days) and Week } 4 \text { (26-32 } \\
\text { days) post discharge }\end{array}$ & $\begin{array}{l}\text { P: No significant difference in self- } \\
\text { reported medication adherence } \\
\text { (Medication Knowledge and Compliance } \\
\text { Scale, data missing to calculate Cohen's } \\
\text { d) between intervention and control } \\
\text { S: No significant increase in medication } \\
\text { knowledge (OR }=1.17 \text { ) between } \\
\text { intervention and control group }\end{array}$ & 7 \\
\hline \multicolumn{7}{|c|}{ Hypertension } \\
\hline $\begin{array}{l}\text { Bosworth et al. } \\
(\underline{56}) \\
\text { USA }\end{array}$ & $\begin{array}{l}\text { Evaluate the } \\
\text { effectiveness of two } \\
\text { SM interventions on } \\
\text { BP control among } \\
\text { patients with } \\
\text { hypertension }\end{array}$ & $\begin{array}{l}\text { Sample size: } 636 \\
\text { Mean Age: } \\
\mathrm{C}+\mathrm{I}_{1-3}: 61 \\
\text { \% Male: } \\
\mathrm{C}+\mathrm{I}_{1-3}: 34 \% \\
\text { Ethnicity: } \\
\mathrm{C}+\mathrm{I}_{1-3}: 49 \% \text { African } \\
\text { American } \\
\text { Income Measure: }\end{array}$ & English & $\begin{array}{l}\text { I: Nurse-led intervention; patients } \\
\text { received: }\left(\mathrm{I}_{1}\right) \text { home BP monitoring } \\
\text { intervention }(\mathrm{BP} \text { measured } 3 \\
\text { times/week) or }\left(\mathrm{I}_{2}\right) \text { telephone- } \\
\text { delivered intervention with low } \\
\text { health literacy materials focused on } \\
\text { social support, self-care behaviors, } \\
\text { and communication with HCP or } \\
\left(\mathrm{I}_{3}\right) \text { behavioral intervention }+\end{array}$ & $\begin{array}{l}\text { P: Significant improvements in the } \\
\text { proportion of patients with adequate BP } \\
\text { control (data missing to calculate Cohen's } \\
\text { D) for the combined intervention grop } \\
\text { S: Significant improvements in systolic } \\
\text { and diastolic BP at } 24 \text { months (data } \\
\text { missing to calculate Cohen's D) in the } \\
\text { combined intervention group }\end{array}$ & 11 \\
\hline
\end{tabular}


Table 1 (continued)

\begin{tabular}{|c|c|c|c|c|c|c|}
\hline & & $\begin{array}{l}\text { Self-reported } \\
\text { financial difficulties } \\
\text { Literacy Measure: } \\
\text { REALM } \\
\mathrm{C}+\mathrm{I}_{1-3}: 27 \% \text { had } \\
\text { literacy level below } \\
\text { the } 9^{\text {th }} \text { grade }(\leq \mathbf{6 0})\end{array}$ & & $\begin{array}{l}\text { home BP monitoring } \\
\text { C: Usual care } \\
\text { Duration of I: } 24 \text { months; patients } \\
\text { in behavioral condition received } \\
\text { bi-monthly phone calls from the } \\
\text { nurse } \\
\text { Timing of measurements: } \\
\text { Baseline, and } 6,12,18 \text { and } 24 \\
\text { months }\end{array}$ & & \\
\hline \multicolumn{7}{|c|}{ Multiple Chronic illnesses } \\
\hline $\begin{array}{l}\text { Gazmararian et } \\
\text { al. (구) } \\
\text { USA }\end{array}$ & $\begin{array}{l}\text { Evaluate a 3-part } \\
\text { intervention } \\
\text { intended to increase } \\
\text { refill adherence } \\
\text { through attention to } \\
\text { health literacy }\end{array}$ & $\begin{array}{l}\text { Sample size: } 275 \\
\text { Mean age: } \\
\text { C: } 52.9 \text {, I: } 40.5 \\
\text { \% Male: } \\
\text { C: } 12.8 \% \text {, I: } 35.3 \% \\
\text { Ethnicity: } \\
\text { C: } 91.9 \% \text {; I: } 89.6 \% \\
\text { African American } \\
\text { Income measure: } \\
\text { area serving a } \\
\text { minority population } \\
\text { Literacy Measure: } \\
\text { REALM C: } 60 \% \text { I: } \\
\text { 58.6\% reading level } \\
\text { below the } 7^{\text {th }} \text { grade }\end{array}$ & $\begin{array}{l}\text { Not } \\
\text { specified } \\
\text { (implicit } \\
\text { English } \\
\text { only) }\end{array}$ & $\begin{array}{l}\text { I: Pharmacist-led intervention } \\
\text { involving telephone-reminders to } \\
\text { fill prescriptions, a "picture } \\
\text { prescription" card and health } \\
\text { communication training for the } \\
\text { pharmacists } \\
\text { C: Other pharmacy } \\
\text { Duration of I: } 6 \text { months } \\
\text { Timing of measurements: } \\
\text { Baseline \& } 6 \text { months }\end{array}$ & $\begin{array}{l}\text { P: No significant improvements in refill } \\
\text { adherence }(d=0.11)\end{array}$ & 5 \\
\hline
\end{tabular}

ED emergency department, HbA1c hemoglobin Alc, HCP healthcare provider, QoL quality of life, SM self-management, SMBG self-monitoring blood glucose. *Effect size was calculated as the mean difference (using the last time point, unless a primary endpoint was specified) of the two study groups divided by the pooled standard deviation of the difference

"low-income" or "urban" community or public health centers. ${ }^{39,42,45,47}$ Three studies identified the target communities as "underserved" 43 or low income. ${ }^{40,57}$ Seven studies reported the level of participant income in dollar amounts, with the majority of annual family incomes being under $\$ 10,000$ USD. ${ }^{38,40,42,44,45,47,57}$

Low literacy defined. Five studies measured literacy using the Rapid Estimate of Adult Literacy in Medicine (REALM) scale, ${ }^{51,53,54,56,57}$ with three of these studies defining low health literacy as a score between 44 and 60 (6th to 9th grade reading level ${ }^{51,54,56}$ ). Three studies utilized the Short Test of Functional Health Literacy in Adults (S-TOFHLA), ${ }^{49,50,52}$ with cutoff scores of less than $22^{49,52}$ or less than $16 .^{50}$ The remaining study used the Test of Functional Health Literacy in Adults (TOFHLA), whereby a score of 0 to 59 constitutes inadequate literacy. ${ }^{55}$

Types of Self-Management Interventions. Intervention duration was variable lasting from a single session to 24 months. Most interventions were delivered face to face by a healthcare professional $(n=17) .^{39,41,42,44-57}$ As detailed in Table 2, interventions were also found to vary in terms of which of the five self-management skills was targeted. Most interventions focused on four to five skills. The most common self-management skill addressed was decision-making $(n=20)$ and partnership with a healthcare provider $(n=19)$. These skills were followed by resource utilization $(n=16)$ and taking action $(n=16)$. Although problem-solving was the least addressed skill, it was still included in 15 studies.

\section{Methodological Quality}

Table 1 provides the methodological quality score of each study (detailed quality assessment in Electronic Supplementary Material S3). Ten studies were assessed to be of high methodological quality, ${ }^{38-40,42,45,46,49,51,54,56}$ seven of moderate methodological quality, ${ }^{35,36,43,48,50,52,55}$ and the remaining six studies were assessed to be of low methodological quality. ${ }^{37,41,44,47,53,57}$

\section{Efficacy of Self-Management Interventions} Efficacy Assessment Based on Self-Management Skills. Of the 23 interventions, 10 were found to be effective (Table 2). Nine of these used problem-solving and eight used taking action and/or resource utilization.

Five Skills. Of the nine interventions that included all five skills, only three were effective on the primary outcomes (Table 2), representing a mix of low income and low health literacy studies. Two of these were of high methodological quality and one of moderate quality. Improved primary outcomes included hemoglobin A1C levels, ${ }^{42}$ dose inhaler technique ${ }^{52}$ asthma symptom-free days, and disease-specific quality of life. ${ }^{46}$

Four Skills. All five interventions utilizing four selfmanagement skills were effective on the primary and secondary outcomes measured, most of which were of moderate-high methodological quality, but including both low income and low health literacy studies. All five effective interventions included taking action, with most of 
Table 2 Self-Management Skills Utilized

\begin{tabular}{|c|c|c|c|c|c|c|c|}
\hline \multirow[t]{2}{*}{ Studies } & \multirow[b]{2}{*}{$\begin{array}{c}\text { Low } \\
\text { income } \\
\text { (LI) } \\
\text { and/or } \\
\text { low health } \\
\text { literacy } \\
\text { (LHL) }\end{array}$} & \multicolumn{5}{|c|}{ Self Management Skills } & \multirow[t]{2}{*}{ Effective } \\
\hline & & $\begin{array}{l}\text { Problem } \\
\text {-Solving }\end{array}$ & $\begin{array}{l}\text { Taking } \\
\text { action }\end{array}$ & $\begin{array}{l}\text { Decision } \\
\text { making }\end{array}$ & Partnership & $\begin{array}{l}\text { Resource } \\
\text { Utilization }\end{array}$ & \\
\hline \multicolumn{8}{|c|}{ FIVE CORE SKILLS } \\
\hline Bennett et al. (노) & $\mathrm{LI}$ & $\mathrm{X}$ & $\mathrm{X}$ & $\mathrm{X}$ & $\mathrm{X}$ & $\mathrm{X}$ & No \\
\hline DeWalt et al. ( $\underline{\underline{50}})$ & LHL & $\mathrm{X}$ & $\mathrm{X}$ & $\mathrm{X}$ & $\mathrm{X}$ & $\mathrm{X}$ & No \\
\hline DeWalt et al. (쑤) & LHL & $\mathrm{X}$ & $\mathrm{X}$ & $\mathrm{X}$ & $\mathrm{X}$ & $\mathrm{X}$ & No \\
\hline Frosch et al. $(\underline{38})$ & LI & $\mathrm{X}$ & $\mathrm{X}$ & $\mathrm{X}$ & $\mathrm{X}$ & $\mathrm{X}$ & No \\
\hline Kiser et al. ( $\underline{52})$ & LHL & $\mathrm{X}$ & $\mathrm{X}$ & $\mathrm{X}$ & $\mathrm{X}$ & $\mathrm{X}$ & Yes \\
\hline Krieger et al. (무) & LI & $\mathrm{X}$ & $\mathrm{X}$ & $\mathrm{X}$ & $\mathrm{X}$ & $\mathrm{X}$ & Yes \\
\hline Martin et al. (44) & LI & $\mathrm{X}$ & $\mathrm{X}$ & $\mathrm{X}$ & $\mathrm{X}$ & $\mathrm{X}$ & No \\
\hline Thom et al. (42) & $\mathrm{LI}$ & $\mathrm{X}$ & $\mathrm{X}$ & $\mathrm{X}$ & $\mathrm{X}$ & $\mathrm{X}$ & Yes \\
\hline Wilson et al. ( $\underline{53})$ & LHL & $\mathrm{X}$ & $\mathrm{X}$ & $\mathrm{X}$ & $\mathrm{X}$ & $\mathrm{X}$ & No \\
\hline \multicolumn{8}{|c|}{ FOUR CORE SKILLS } \\
\hline Bosworth et al. ( $\underline{56})$ & $\mathrm{LI}+\mathrm{LHL}$ & & $\mathrm{X}$ & $\mathrm{X}$ & $\mathrm{X}$ & $\mathrm{X}$ & Yes \\
\hline Cavanaugh et al. (54) & LHL & $\mathrm{X}$ & $\mathrm{X}$ & $\mathrm{X}$ & $\mathrm{X}$ & & Yes \\
\hline Fischer et al. (37) & LI & $\mathrm{X}$ & $\mathrm{X}$ & & $\mathrm{X}$ & $\mathrm{X}$ & Yes \\
\hline Gregg et al. (39) & $\mathrm{LI}$ & $\mathrm{X}$ & $\mathrm{X}$ & $\mathrm{X}$ & $\mathrm{X}$ & & Yes \\
\hline Riley et al. (47) & $\mathrm{LI}$ & $\bar{X}$ & $\mathrm{X}$ & $\mathrm{X}$ & & $\mathrm{X}$ & Yes \\
\hline \multicolumn{8}{|c|}{ THREE CORE SKILLS } \\
\hline Fischer et al. ( $\underline{36})$ & LI & & $\mathrm{X}$ & & $\mathrm{X}$ & $\mathrm{X}$ & Mixed* \\
\hline $\begin{array}{l}\text { Gazmararian et al. } \\
(\underline{57})\end{array}$ & $\mathrm{LI}+\mathrm{LHL}$ & & & $\mathrm{X}$ & $\mathrm{X}$ & $\mathrm{X}$ & No \\
\hline Piette et al. (무) & LI & $\mathrm{X}$ & & $\mathrm{X}$ & & $\mathrm{X}$ & Yes \\
\hline Rudd et al. (묘) & LHL & $\mathrm{X}$ & & & $\mathrm{X}$ & $\mathrm{X}$ & Yes \\
\hline \multicolumn{8}{|c|}{ TWO CORE SKILLS } \\
\hline Arora et al. (35) & $\mathrm{LI}$ & & $\mathrm{X}$ & $\mathrm{X}$ & & & No \\
\hline Cordasco et al. (55) & $\mathrm{LI}+\mathrm{LHL}$ & & & $\mathrm{X}$ & $\mathrm{X}$ & & No \\
\hline Kripalanni et al. (51) & LHL & & & $\mathrm{X}$ & $\mathrm{X}$ & & No \\
\hline Sequeira et al. (41) & $\mathrm{LI}$ & & & $\mathrm{X}$ & $\mathrm{X}$ & & No \\
\hline \multicolumn{8}{|c|}{ ONE CORE SKILL } \\
\hline Bove et al. (며) & LI & & & $\mathrm{X}$ & & & No \\
\hline
\end{tabular}

Note: Definitions of core skills: (a) Problem solving: the ability to define the problem, generate and implement solutions, and evaluate outcomes; (b) taking action: involves the creation of action plans and the development of self-efficacy; (c) decision-making: includes self-monitoring and responding to changing disease condition; (d) partnership: involves forming an ongoing client-healthcare provider partnership; (e) resource utilization: the ability to seek out and use multiple healthpromoting resources. *Four interventions were tested in this study, and significant outcomes were noted for the enhanced provider PRC treatment group

them also including problem-solving or partnership (Table 2). Primary outcomes targeted were blood pressure, ${ }^{56}$ hemoglobin $\mathrm{A} 1 \mathrm{C},{ }^{39,54}$ lipids, ${ }^{37}$ and socioenvironmental resources. ${ }^{47}$ Secondary outcomes were self-efficacy, ${ }^{54}$ cost to the healthcare system, ${ }^{37}$ acceptance, ${ }^{39}$ self-management behavior, ${ }^{39}$ medication adherence, ${ }^{47}$ and minutes of physical activity. ${ }^{47}$

Three Skills. Two of the four studies targeting three selfmanagement skills were effective $e^{40,48}$; these were the only 
interventions that included problem-solving and resource utilization. No pattern was noted across the low income versus low health literacy studies. Primary outcomes favorably affected by the interventions were self-efficacy, ${ }^{40}$ depression, ${ }^{40}$ days spent in bed because of illness, ${ }^{40}$ and self-efficacy. ${ }^{48}$ The only intervention that was not effective according to Gazmararian et al. ${ }^{57}$ was the only one to omit problem-solving or taking action.

Two or One Skill(s). None of the interventions utilizing one or two self-management skills were effective. No pattern was noted across the low income versus low health literacy studies.

Efficacy Assessment by Outcomes. There was no consistency across studies regarding the outcomes favored, with many outcomes only measured in 1-2 studies. Those measured in at least four analyses are summarized; the remaining analyses are presented in Table 1. Of note, as no efficacy patterns were noted across the low income and low health literacy studies, these are presented together. The most frequently measured outcome was blood pressure; however, only $4^{36,56}$ of the 16 analyses were significant. A1C was the next most frequent outcome, with $5^{36,39,42,54}$ of the 13 analyses found to be significant. Lipids were included in 12 analyses, whereby $2^{37}$ were significant. Of the nine analyses focusing on medication adherence, only one $\mathrm{e}^{47}$ was significant. For health behaviors, one ${ }^{47}$ out of eight analyses was significant. None of the six analyses reporting healthcare utilization were significant. ${ }^{37,46,49,50}$ One $^{50}$ out of five analyses was significant for knowledge. For selfcare and self-management, two ${ }^{39,52}$ out of the seven analyses were significant. A more positive result was found for empowerment, with four ${ }^{40,48,50,54}$ of the five analyses reporting significant effects. Similarly, two ${ }^{46,49}$ of the four studies for disease-specific quality of life were significant. Based on this review, only two outcomes met the criterion of being effective in at least $50 \%$ of the analyses: empowerment or self-efficacy and disease-specific quality of life.

Efficacy Based on Tailoring. Ten interventions ${ }^{36,38,40,44-}$ $47,50,53,57$ tailored their content, and only three (all with individuals of low income) were effective. ${ }^{40,46,47}$ No pattern in terms of skills targeted was noted.

\section{DISCUSSION}

This review sought to critically appraise the empirical evidence on the efficacy of self-management interventions in a population of low-income and/or low health literate adults diagnosed with a chronic physical illness. A thorough understanding of the components of effective self-management interventions is essential given the increased number of underserved patients living with a chronic illness. ${ }^{26}$

According to the 2015 United States federal poverty guidelines, low income is defined as a family income of $\$ 24,250$ USD annually or less for four people living in the same residence. ${ }^{58}$ Fifteen of the 16 studies that examined low-income populations failed to report the participant household income relative to federal poverty guidelines. Instead, the authors reported the use of self-management interventions in "federally qualified health centers," "safety net clinics," or low-income clinics or neighborhoods. This assumes homogeneity of the population regarding socioeconomic status. According to Darnell, ${ }^{59}$ safety net clinics may service both low income and uninsured populations. As such, based on the studies reviewed, drawing meaningful conclusions about the efficacy of selfmanagement interventions within a low-income population presented some challenges. This is in contrast to all studies $(n=10)$ examining a population of low health literate adults using a validated measure of health literacy.

Reviews of self-management interventions have further found that face-to-face contact between participants and intervention leaders is associated with better outcomes. ${ }^{18,60}$ The present review did not note any patterns potentially linking the mode of delivery or the person implementing the intervention to efficacy. Similarly, although Battersby et al. ${ }^{60}$ found that numerous intervention sessions yielded better outcomes, this pattern was not noted and both single and multi-session interventions yielded positive outcomes in the present review. Other reviews have also found that chronic illness typology may moderate the efficacy of self-management interventions. ${ }^{18,60,61}$ The present review found no discernable pattern across illness type, which may be due to the limited research on self-management in chronically ill populations with low income and/or low health literacy.

The current review found that proportionally interventions using three or four self-management skills were more effective than those presenting less than three or five skills. This likely relates to the multifaceted nature of these interventions through the use of social, behavioral, and cognitive approaches to improve disease management. ${ }^{10}$ However, participants in interventions with five skills might have felt overwhelmed and/or might not have been given enough time to develop all skills. While the use of four skills appears promising, economic constraints and/or a lack of resources may hinder the development and implementation of multifaceted self-management interventions. If only one self-management skill can be targeted, findings of the current review would suggest problem-solving, as more than half of the 15 interventions with problem-solving were effective on primary outcomes and two additional studies found significant changes in secondary outcome measures. ${ }^{49,50}$ According to Savery, ${ }^{62}$ problem-solving skills help in the development of higherorder thinking. Studies examining the use of problem-based learning have shown that such an approach enables learners to identify and understand the important elements of the 
situation, develop an understanding of the relationship between elements, and facilitates improved decision-making, communication, and collaboration. ${ }^{62-64}$ This is likely mediated through improvements in the client's engagement, knowledge, confidence, skills, and commitment to making optimal health adjustments. ${ }^{65}$ Arguably, the development of problemsolving skills is implicitly linked to improvements in the other four self-management skills. As such, it has the most potential for influencing behavioral, cognitive, and social health changes. In support of this, many studies have found that problem-solving is a key component of effective selfmanagement across various chronic conditions. ${ }^{66,67}$ In addition to problem-solving, two other self-management skills were found to be effective in half of the interventions that used them: taking action and resource utilization.

In terms of efficacy of the interventions reviewed on healthrelated outcomes, this review highlighted that selfmanagement interventions among individuals with a chronic illness with low health literacy and/or low income may benefit in terms of enhanced empowerment (or self-efficacy) and disease-specific quality of life. Although a central aim of self-management interventions is to increase participants' self-efficacy to carry out a behavior, ${ }^{10}$ only five interventions measured this outcome. For many outcomes considered, less than $50 \%$ of the analyses were not significant. Partially, this finding might be explained by the reliance on mostly 'distal' outcomes (e.g., lipids, blood pressure), which depend on a number of factors that are not directly influenced by the interventions. ${ }^{68}$ Reliance on proximal outcomes or outcomes that can be directly affected by the interventions' content and goals might have been more appropriate. ${ }^{68}$

Tailoring was another feature considered in the present review, as it is hypothesized to increase the relevance, interest, and use of interventions. ${ }^{69,70}$ In general, the process of tailoring prunes out the superfluous or irrelevant information and highlights only what individuals would find most pertinent. ${ }^{69,70}$ Tailored print- ${ }^{71,72}$ and web-based ${ }^{73}$ interventions have been found to be more effective than non-tailored approaches. Contrary to these results, less than $50 \%$ of interventions with tailored content were found to be overall effective.

\section{Strengths and Limitations}

Methods are described in great detail to enhance reproducibility. However, one limitation is that several studies did not explicitly explain the core components of their selfmanagement intervention. However, to reduce bias, five reviewers independently assessed each intervention to identify its self-management skills. Another limitation is the low methodological quality of some studies. Also, for many outcomes and some illnesses the number of analyses was small, and findings should be interpreted with caution. Furthermore, mental health comorbidities were typically not documented across studies, which precludes any subgroup analyses.

\section{Implications for Future Research}

Further inquiry is warranted regarding the use of problemsolving, taking action and resource utilization in selfmanagement interventions, and determining which combination of these three skills result in the highest efficacy. In addition, measuring both proximal and distal outcomes in future studies might assist in further determining those interventions that are most effective for what type of outcome. Future studies would also benefit from some consensus on the type of outcomes that should be measured. Replication studies outside the US are also needed.

\section{CONCLUSION}

Low income and low health literacy are both associated with poorer health outcomes and higher rates of chronic disease. Thus, self-management represents an important healthcare strategy for these populations. This systematic review described the current use of self-management interventions in populations with low income or low health literacy and synthesized their efficacy. Overall, the current review found that effective interventions tended to focus on problem-solving and to a certain extent taking action and resource utilization. A wide range of health-related outcomes were considered, but only empowerment (or self-efficacy) and disease-specific quality of life were found to be positively affected by the interventions. Tailoring did not seem to impact on efficacy. Future high-quality trials further evaluating problem-solving in combination with taking action and resource utilization among individuals with low health literacy and/or income and replication studies outside the US are needed.

Acknowledgements: We wish to acknowledge Drs. Lisa Merry and Argerie Tsimicalis of McGill University for their guidance and support in the preparation of this systematic review. Preliminary results were presented in the form of an e-poster in a Graduate level class on research methods in Nursing at McGill University, and an abstract was presented (poster presentation) at the Health Literacy Annual Research Conference (HARC) in October 2016 (title: The effectiveness of tailoring self-management interventions for individuals with low health literacy or low income: A systematic review). There has been no significant financial support for this work that could have influenced its outcome. Sylvie Lambert was supported by a Canada Research Chair (Tier 2).

Corresponding Author: Sylvie D. Lambert, $R N, B S c(N), P h D$; Ingram School of Nursing, McGill University, Quebec, Montreal, Canada (e-mail: sylvie.lambert@mcgill.ca).

\section{Compliance with Ethical Standards:}

Conflict of Interest: The authors declare that they do not have a conflict of interest.

\section{REFERENCES}

1. Wu S-Y, Green A. Projection of chronic illness prevalence and cost inflation. Santa Monica: RAND Health 2000, 18. 
2. Walker D, Adebajo A, Heslop P, et al. Patient education in rheumatoid arthritis: the effectiveness of the ARC booklet and the mind map. Rheumatology (Oxford). 2007, 46:1593-1596.

3. Vogeli C, Shields AE, Lee TA, et al. Multiple chronic conditions: prevalence, health consequences, and implications for quality, care management, and costs. J Gen Intern Med 2007, 22:391-395.

4. Berkman ND, Sheridan SL, Donahue KE, Halpern DJ, Crotty K. Low health literacy and health outcomes: an updated systematic review. Ann Intern Med 2011, 155:97-107.

5. Davy C, Bleasel J, Liu H, et al. Effectiveness of chronic care models: opportunities for improving healthcare practice and health outcomes: a systematic review. BMC Health Serv Res 2015, 15:194.

6. Coleman K, Austin BT, Brach C, Wagner EH. Evidence on the Chronic Care Model in the new millennium. Health affairs (Milwood). 2009, 28:75-85

7. Davy C, Bleasel J, Liu H, et al. Effectiveness of chronic care models: opportunities for improving healthcare practice and health outcomes: a systematic review. BMC Health Serv Res 2015, 15:194.

8. Bodenheimer $\mathbf{T}$, Wagner $\mathbf{E H}$, Grumbach $\mathbf{K}$. Improving primary care for patients with chronic illness. JAMA 2002, 288:1775-1779.

9. Adams K, Greiner AC, Corrigan JM. 1st Annual Crossing the Quality Chasm Summit: A focus on communities. Washington DC: National Academies Press, 2004.

10. Lorig KR, Holman HR. Self-management education: History, definition, outcomes, and mechanisms. Ann Behav Med 2003, 26:1-7.

11. Beatty L, Lambert S. A systematic review of internet-based self-help therapeutic interventions to improve distress and disease-control among adults with chronic health conditions. Clin Psychol Rev 2013, 33:609-622.

12. Boger EJ, Demain S, Latter S. Self-management a systematic review of outcome measures adopted in self-management interventions for stroke. Disabil Rehabil 2013, 35:1415-1428.

13. Dorn SD. Systematic review: self-management support interventions for irritable bowel syndrome. Aliment Pharmacol Ther 2010, 32:513-521.

14. Du S, Yuan C, Xiao $\mathbf{X}$, et al. Self-management programs for chronic musculoskeletal pain conditions: a systematic review and meta-analysis. Patient Educ Couns 2011, 85:e299-e310.

15. Plow MA, Finlayson M, Rezac M. A scoping review of self-management interventions for adults with multiple sclerosis. PM\&R 2011, 3:251-262.

16. Barlow J, Wright C, Sheasby J, Turner A, Hainsworth J. Selfmanagement approaches for people with chronic conditions: a review. Patient Educ Couns 2002, 48:177-187.

17. Eichner J, Blumenthal D. Medicare in the 21st Century: Building a Better Chronic Care System. National Academy of Social Insurance; 2003. https:// doi.org/10.2139/ssrn.555632.

18. Warsi A, Wang PS, LaValley MP, Avorn J, Solomon DH. Selfmanagement education programs in chronic disease: a systematic review and methodological critique of the literature. Arch Intern Med 2004, 164:9-23.

19. Meraviglia M, Stuifbergen A, Parsons D, Morgan S. Health promotion for cancer survivors: adaptation and implementation of an intervention. Holist Nurs Pract 2013, 27:140-147.

20. Walker D, Adebajo A, Heslop P, et al. Patient education in rheumatoid arthritis: the effectiveness of the ARC booklet and the mind map. Rheumatology 2007, 46:1593-1596.

21. Canadian Public Health Association. Ottawa Charter for Health Promotion. Can J Public Health. 1986; 77:425-430.

22. DeNavas-Walt C, Proctor BD, Smith JC. US Census Bureau, Current population reports, P60-245, income, poverty, and health insurance coverage in the United States: 2012, US Government Printing Office, Washington, DC, 2013.

23. Betancourt MT, Roberts KC, Bennett TL, et al. Monitoring chronic diseases in Canada: the Chronic Disease Indicator Framework. Chronic Dis Inj Can 2014, 34:1-30.

24. Dubay LC, Lebrun LA. Health, behavior, and health care disparities: disentangling the effects of income and race in the United States. Int J Health Serv 2012, 42:607-625.

25. World Health Organization. Noncommunicable diseases. Retrieved October 2, 2015 from http://www.who.int/mediacentre/factsheets/fs355/en/

26. Mikkonen J, Raphael D. Social determinants of health: The Canadian facts. Toronto: York University School of Health Policy and Management. 2010. Available at http://thecanadianfacts.org/The_Canadian_Facts. pdf.

27. Kutner M, Greenburg E, Jin Y, Paulsen C. The Health Literacy of America's Adults: Results from the 2003 National Assessment of Adult Literacy (NCES 2006-483). U.S. Department ofEducation. Washington, DC: National Center for Education Statistics. 2006.
28. Rootman I, Gordon-E1-Bihbety D. A vision for a health literate Canada report of the Expert Panel on Health Literacy. Ottawa: Canadian Public Health Association, 2008.

29. Moher D, Liberati A, Tetzlaff J, Altman DG. Group TP Preferred reporting items for systematic reviews and meta-analyses: The PRISMA statement Ann Intern Med 2009, 151:264-269.

30. Higgins JP, Green S. Cochrane handbook for systematic reviews of interventions version 5.0.0 [updated February 2008]. The Cochrane Collaboration. 2008. Available: http://www.cochrane-handbook.org/. Accessed 15 February 2016.

31. Chambless DL, Hollon SD. Defining empirically supported therapies. J Consult Clin Psychol 1998, 66:7-18.

32. Blundell M. Understanding and synthesizing my numerical data. In A. Boland, M. G. Cherry and R. Dickson (eds), Doing a systematic review—A student's guide. Los Angeles, Sage, 2014.

33. Bos-Touwen I, Jonkman N, Westland H, et al. Tailoring of selfmanagement interventions in patients with heart failure. Current Heart Failure Reports, 2015, 12(3):223-235. https://doi.org/10.1007/s11897015-0259-3.

34. Cohen JH. Statistical power analysis for the behavioral sciences (2nd Ed.). Hillsdale: Lawrence Earlbaum Associates, 1988.

35. Arora S, Peters AL, Burner E, Lam CN, Menchine M. Trial to examine text message-based mhealth in emergency department patients with diabetes (TExT-MED): A randomized controlled trial. Ann Emerg Med. 2014, 63:745-754.e746.

36. Fischer HH, Eisert SL, Durfee MJ, et al. The impact of tailored diabetes registry report cards on measures of disease control: a nested randomized trial. BMC Med Inform Decis Mak 2011, 11:12.

37. Fischer HH, Moore SL, Ginosar D, et al. Care by cell phone: text messaging for chronic disease management. Am J Manag Care 2012, 18:e42-47.

38. Frosch DL, Uy V, Ochoa S, Mangione CM. Evaluation of a behavior support intervention for patients with poorly controlled diabetes. Arch Intern Med 2011, 171:2011-2017.

39. Gregg JA, Callaghan GM, Hayes SC, Glenn-Lawson JL. Improving diabetes self-management through acceptance, mindfulness, and values: a randomized controlled trial. J Consult Clin Psychol 2007, 75:336-343.

40. Piette JD, Weinberger M, McPhee SJ. The effect of automated calls with telephone nurse follow-up on patient-centered outcomes of diabetes care: a randomized, controlled trial. Med Care 2000, 38:218-230.

41. Sequeira PA, Montoya L, Ruelas V, et al. Continuous glucose monitoring pilot in low-income type 1 diabetes patients. Diabetes Technol Ther 2013, 15:855-858.

42. Thom DH, Ghorob A, Hessler D, et al. Impact of peer health coaching on glycemic control in low-income patients with diabetes: a randomized controlled trial. Ann Fam Med 2013, 11:137-144.

43. Bove AA, Homko C, Santamore W, et al. Managing hypertension in urban underserved subjects using telemedicine: A clinical trial. J Am Coll Cardiol 2013, 1:E1401

44. Martin MY, Kim Y, Kratt P, et al. Medication adherence among rural, low-income hypertensive adults: A randomized trial of a multimedia community-based intervention. Am J Health Promot 2011, 25:372-378.

45. Bennett GG, Warner ET, Glasgow RE, et al. Obesity treatment for socioeconomically disadvantaged patients in primary care practice. Arch Intern Med 2012, 172:565-574

46. Krieger J, Song L, Philby M. Community health worker home visits for adults with uncontrolled asthma-the HomeBase trial randomized clinical trial. JAMA Intern Med 2015, 175:109-117.

47. Riley KM, Glasgow RE, Eakin EG. Resources for health: A socialecological intervention for supporting self-management of chronic conditions. J Health Psychol 2001, 6:693-705.

48. Rudd RE, Blanch DC, Gall V, et al. A randomized controlled trial of an intervention to reduce low literacy barriers in inflammatory arthritis management. Patient Educ Couns 2009, 75:334-339.

49. Dewalt DA, Schillinger D, Ruo B, et al. Multisite randomized trial of a single-session versus multisession literacy-sensitive self-care intervention for patients with heart failure. Circulation 2012, 125:2854-2862.

50. DeWalt DA, Malone RM, Bryant ME, et al. A heart failure selfmanagement program for patients of all literacy levels: a randomized, controlled trial [ISRCTN11535170]. BMC Health Serv Res 2006, 6:30.

51. Kripalani S, Schmotzer B, Jacobson TA. Improving Medication Adherence through Graphically Enhanced Interventions in Coronary Heart Disease (IMAGE-CHD): a randomized controlled trial. J Gen Intern Med 2012, 27:1609-1617. 
52. Kiser $\mathbf{K}$, Jonas $\mathbf{D}$, Warner $\mathbf{Z}$, et al. A randomized controlled trial of a literacy-sensitive self-management intervention for chronic obstructive pulmonary disease patients. J Gen Intern Med 2012, 27:190-195.

53. Wilson FL, Mood D, Nordstrom CK, Risk J. The effect of low literacy on the self-care behaviors of men receiving radiation therapy. Nurs Sci Q. 2010, 23:326-333.

54. Cavanaugh $\mathbf{K}$, Wallston KA, Gebretsadik T, et al. Addressing literacy and numeracy to improve diabetes care: two randomized controlled trials. Diabetes Care 2009, 32:2149-2155.

55. Cordasco KM, Asch SM, Bell DS, et al. A low-literacy medication education tool for safety-net hospital patients. Am J Prev Med 2009, 37:S209-S216.

56. Bosworth HB, Olsen MK, Grubber JM, et al. Two self-management interventions to improve hypertension control: a randomized trial. Ann Intern Med. 2009, 151:687-695.

57. Gazmararian J, Jacobson KL, Pan Y, Schmotzer B, Kripalani S. Effect of a pharmacy-based health literacy intervention and patient characteristics on medication refill adherence in an urban health system. Ann Pharmacother 2010, 44:80-87.

58. US Department of Health and Human Services. The Poverty Guidelines Updated Periodically in the Federal Register. Washington, DC: US Department of Health and Human Services. 2015, 42.

59. Darnell JS. Free clinics in the United States: a nationwide survey. Arch Intern Med 2010, 170:946-953.

60. Battersby $\mathbf{M}$, Von Korff $\mathbf{M}$, Schaefer J, et al. Twelve evidence-based principles for implementing self-management support in primary care. Jt Comm J Qual Patient Saf 2010, 36:561-570.

61. Nolte S, Osborne RH. A systematic review of outcomes of chronic disease self-management interventions. Qual Life Res 2013, 22:1805-1816.

62. Savery JR. Overview of problem-based learning: Definitions and distinctions. Interdisc J Probl-Based Learn 2006, 1:9-20.

63. King DK, Glasgow RE, Toobert DJ, et al. Self-efficacy, problem solving, and social-environmental support are associated with diabetes selfmanagement behaviors. Diabetes Care 2010, 33:751-753.
64. Primožič S, Avbelj M, Dernovšek MZ, Oblak MR. Specific cognitive abilities are associated with diabetes self-management behavior among patients with type 2 diabetes. Diabetes Res Clin Pract 2012, 95:48-54.

65. Auerbach JA, Krimgold BK. Income, socioeconomic status, and health exploring the relationships. Washington DC: National Policy Association, 2001.

66. Glasgow RE, Fisher L, Skaff M, Mullan J, Toobert DJ. Problem solving and diabetes self-management investigation in a large, multiracial sample. Diabetes Care 2007, 30:33-37.

67. Hill-Briggs F, Yeh H-C, Gary TL, et al. Diabetes problem-solving scale development in an adult, African American sample Diab Educ 2007, 33:291-299.

68. Osborne R, Elsworth G, Whitfield $\mathbf{K}$. The Health Education Impact Questionnaire (heiQ): an outcomes and evaluation measure for patient education and self-management interventions for people with chronic conditions. Patient Educ Couns 2007, 66:192-201.

69. Campbell MK, Guintiliani LM. Tailored interventions in public health Where does tailoring fit in interventions to reduce health disparities? Am Behav Sci 2006, 49: 1-19.

70. Kreuter M, Strecher V, Glassman B. One size does not fit all: The case for tailoring print materials. Ann Behav Med 1999, 21:276-283.

71. Short CE, James EL, Plotnikoff RC, Girgis A. Efficacy of tailored-print interventions to promote physical activity: a systematic review of randomised trials. Int $\mathrm{J}$ Behav Nutr Phys Act 2011, 8:113.

72. Noar SM, Benac CN, Harris MS. Does tailoring matter? Meta-analytic review of tailored print health behavior change interventions. Psychol Bull 2007, 133:673-693.

73. Lin ZC, Effken JA. Effects of a tailored web-based educational intervention on women's perceptions of and intentions to obtain mammography. J Clin Nurs 2010, 19:1261-1269. 